Illinois State University

ISU ReD: Research and eData

Theses and Dissertations

3-24-2021

\title{
A Computational Investigation Of The Biophysical Mechanisms Underlying Thermotaxis In The Afd Neurons Of Caenorhabditis Elegans
}

Zachary Mobille

Illinois State University, zdm428@gmail.com

Follow this and additional works at: https://ir.library.illinoisstate.edu/etd

Part of the Applied Mathematics Commons, Biology Commons, and the Biophysics Commons

\section{Recommended Citation}

Mobille, Zachary, "A Computational Investigation Of The Biophysical Mechanisms Underlying Thermotaxis In The Afd Neurons Of Caenorhabditis Elegans" (2021). Theses and Dissertations. 1391.

https://ir.library.illinoisstate.edu/etd/1391

This Thesis is brought to you for free and open access by ISU ReD: Research and eData. It has been accepted for inclusion in Theses and Dissertations by an authorized administrator of ISU ReD: Research and eData. For more information, please contact ISUReD@ilstu.edu. 


\section{A COMPUTATIONAL INVESTIGATION OF THE BIOPHYSICAL MECHANISMS UNDERLYING THERMOTAXIS IN THE AFD NEURONS OF \\ CAENORHABDITIS ELEGANS}

\section{ZACHARY MOBILLE}

\section{Pages}

Thermotaxis in the nematode Caenorhabditis elegans (C. elegans) is studied at the cellular scale of the amphid finger-like ciliated (AFD) neurons, which have previously been shown to be essential for thermoreception. The electrochemical responses of AFD during temperature stimuli are described with ordinary differential equations. The chemical quantity of interest is the concentration of free calcium ions, while the electrical one is the membrane current. The primary calcium model is a modified version of that published by Kuramochi and Doi in 2017 to explain the calcium responses of the chemosensitive amphid single-ciliated right (ASER) neuron to fluctuations in extracellular salt concentration. To account for the effects of temperature, changes to the stimuli conditions under which inactivation takes place are made and two temperature-sensitive Arrhenius parameters are added. This model is validated using experimental data from Clark et al. (2006) and Yoshida et al. (2016). The AFD membrane currents during a voltage-clamp are also modeled mathematically, and are found to be described by three simple linear equations that are analytically tractable. The membrane current model is analyzed and validated using experimental data from Ramot et al. (2008). Three parameters in this original model modulate the temperature sensitivity, time scale, and monotonicity of the current response during a voltage clamp. This work may serve as a precursor to more advanced modeling studies incorporating voltage and calcium of the AFD neuron as coupled temperature response variables. It may also play a role in guiding future fluorescence and/or patch-clamp experiments of the AFD neuron with the goal of quantifying its thermal sensitivity.

KEYWORDS: AFD neurons, C. elegans, thermotaxis, mathematical modeling, single-cell biophysics 


\section{A COMPUTATIONAL INVESTIGATION OF THE BIOPHYSICAL MECHANISMS UNDERLYING THERMOTAXIS IN THE AFD NEURONS OF \\ CAENHORABDITIS ELEGANS}

ZACHARY MOBILLE

A Thesis Submitted in Partial Fulfillment of the Requirements for the Degree of MASTER OF SCIENCE

Department of Mathematics ILLINOIS STATE UNIVERSITY 
(C) 2021 Zachary Mobille 


\section{A COMPUTATIONAL INVESTIGATION OF THE BIOPHYSICAL MECHANISMS UNDERLYING THERMOTAXIS IN THE AFD NEURONS OF \\ CAENHORABDITIS ELEGANS}

ZACHARY MOBILLE

COMMITTEE MEMBERS:

Epaminondas Rosa, Co-Chair

Olcay Akman, Co-Chair

Andrés Vidal-Gadea

Papa Sissokho 


\section{ACKNOWLEDGMENTS}

There are several parties I would like to recognize as integral contributors to this work. WormBase.org, WormBook.org, and WormAtlas.org have all been essential resources to me in learning about the basics of $C$. elegans. All items originally authored by those associated with these online resources are not my own, and cited where used here. Chance Bainbridge and Dr. Andrés VidalGadea significantly improved my understanding of the nervous system and behavior of $C$. elegans through many helpful conversations. It was a public talk by Andrés at ISU that originally peaked my interest in the critter. I thank Dr. Olcay Akman for awarding me studentship in the biomath program and a great environment to conduct interdisciplinary research in. Dr. Papa Sissokho was my superb teacher for differential equations, which is a subdiscipline of mathematics that forms the quantitative foundations of this project. The ISU physics and math departments, at large, have given me settings to learn and grow over the past 6 years. I am immensely thankful for the services

and relationships they have provided me. I am additionally grateful for the welcoming nature of both the School of Information Technology as well as the School of Biological Sciences at ISU, who have allowed me in to their classes when disguised as an aspiring physicist. In particular, Drs. Wolfgang Stein and Paul Garris let me in to their neurobiology seminar while studying physics as an undergraduate. It was here where I first had the chance to talk extensively with neuroscientists about their subject matter, which gave me invaluable perspectives on the dynamics of biological systems. Dr. George Rutherford showed me the magic of electronics and led me to the realization that engineering and science aren't as distinct as we may often think.

Of special mention are Drs. Rosangela Follmann and Epaminondas Rosa, whose support and mentorship have been essential throughout the past few years of my academic career. I will be forever thankful for them sharing their passion for research and teaching with me. The beautiful subject of computational neuroscience would likely be unknown to me today, if it weren't for Dr. Rosa.

Lastly, I thank my parents Janet \& Jacques Mobille for their love, support, and presence throughout my life. They have enabled me to pursue what interests and challenges me, which I will always remember.

Z.M. 


\section{CONTENTS}

Page

ACKNOWLEDGMENTS

CONTENTS

FIGURES

CHAPTER I: INTRODUCTION 1

$\begin{array}{ll}\text { Historical context } & 1\end{array}$

$\begin{array}{ll}\text { Motivations } & 3\end{array}$

$\begin{array}{ll}\text { Subject of Study } & 3\end{array}$

CHAPTER II: INTRACELLULAR CALCIUM DYNAMICS 6

$\begin{array}{ll}\text { Model Equations } & 6\end{array}$

Single Response at Constant Temperature $\quad 8$

$\begin{array}{ll}\text { Matching the Experimental Data } & 16\end{array}$

Summary of the $\mathrm{Ca}^{2+}$ Model Results 21

CHAPTER III: MEMBRANE ELECTRODYNAMICS 22

Model Equations with Constant Voltage Solution 22

Properties of the Potassium Activation during a Voltage Clamp 26

Using the Steady-State Current to Match the Data 29

CHAPTER IV: CONCLUSIONS

$\begin{array}{ll}\text { Calcium } & 33\end{array}$

$\begin{array}{ll}\text { Membrane Electrodynamics } & 34\end{array}$

Broad Conclusions, Caveats, and Future Outlook 36

$\begin{array}{ll}\text { REFERENCES } & 38\end{array}$

APPENDIX A: VARIABLES $W_{d}$ AND $W_{a}$ (ADAPTED FROM [25]) 43

APPENDIX B: GOLDBETER-DUPONT-BERRIDGE CA ${ }^{2+}$ MODEL 44

APPENDIX C: FAST INACTIVATION POTASSIUM CURRENT MODEL 46

APPENDIX D: NUMERICAL DETAILS AND STATEMENT OF ACCESS 49 


\section{FIGURES}

Figure $\quad$ Page

1. A microscope image of $C$. elegans 2

2. Schematic representation of the AFDR neuron $\quad 5$

3. Schematic representation of the AFDL neuron 5

4. Illustration of the sensory endings of AFD. Copyright 2002-2021 WormAtlas 5

5. Dendritic calcium response of simulated AFD neuron 8

6. Somatic calcium response of simulated AFD neuron 9

7. Axonal calcium response of simulated AFD neuron 9

8. Normalized response for three different $\phi_{0}$ values, in warm conditions 11

9. Normalized response for three different $\rho_{0}$ values, in warm conditions 11

10. Normalized response for three different $\phi_{0}$ values, in cold conditions 12

11. Normalized response for three different $\rho_{0}$ values, in cold conditions 12

12. Peak time of $c_{d}$ during a 60 -second pulse for discrete $\phi_{0}$ and $T \quad 13$

13. Peak time of $c_{d}$ during a 60 -second pulse for discrete $\rho_{0}$ and $T \quad 13$

14. Peak time of $c_{d}$ during a 60-second pulse for continuous $\phi_{0}$ and $T \quad 14$

15. Peak time of $c_{d}$ during a 60-second pulse for continuous $\rho_{0}$ and $T \quad 14$

16. Simulated response to oscillating temperature 16

17. Experimental data from figure 1B (bottom) of Clark et al. (2006) 17

18. Simulated response to linear temperature ramp below $T_{C} \quad 18$

19. Experimental data from figure 1B (top) of Clark et al. (2006) 19

20. Axonal response of simulated AFD neuron to a linear ramping temperature above $T_{C} \quad 20$

21. Experimental data from figure 1B of Yoshida et al. (2016) 21

22. Circuit model of the AFD neuronal membrane 22

23. Equation 18 plotted for various values of $\rho \quad 26$

24. Equation 18 plotted for various values of $c_{0} \quad 28$

25. Equation 18 plotted for various values of $m_{0} \quad 28$

26. Experimental data from figure 3A of Ramot et al. (2008) 30

27. Theoretical current responses, $T=23.6^{\circ} \mathrm{C}$ 
28. Theoretical current responses, $T=17.7^{\circ} \mathrm{C}$

29. Relationship between initial $c_{0}, V$, and $T$

30. Local network of AFD with its synaptic partners

31. Simulation of minimal model for $\mathrm{Ca}^{2+}$ oscillations

32. Simulation of equations $30-33$

33. Experimental data from figure 1 of Ramot et al. (2008) 


\section{CHAPTER I: INTRODUCTION}

\section{Historical context}

Caenorhabditis elegans is a transparent nematode approximately $1 \mathrm{~mm}$ in length often found inhabiting rotting fruits and vegetables in humid temperate climates across the Earth. Its genus is derived from Greek and Latin: "Caeno" means "recent" and "rhabditis" means "rod" (referring to the approximately-cylindrical shape of the creature). The species name "elegans" is related to the English word "elegant", used here as a descriptor of the graceful manner in which this nematode swims. There exists a male and also a hermaphrodite; this work is focused on the hermaphrodite. The terms "C. elegans" and "the worm" will be used interchangeably throughout when referring to this organism. The free-living nematode genus Rhabditis, to which C. elegans belongs, was proposed as a specimen worth studying in a publication by Ellsworth C. Dougherty and Hermione Grant Calhoun of the University of California, Berkeley, in 1948. They identified Rhabditis as an ideal target for studying genetics and development due to its short life span, small number of cells (which is fairly consistent from individual to individual), and the relative ease with which it can be cultivated in the lab [10].

Dougherty was a practicing physician and nematologist who was motivated by the prospect of applying the recently-developed biochemical methods of his time to understanding the genetics and subsequent physiology of multicellular organisms. He was particularly interested in the hermaphroditic C. elegans because of the minimal genetic variability inherent to such a selfreproducing species. Dougherty introduced Sydney Brenner, a molecular biologist working in Cambridge at the time, to C. Elegans in a letter signed October 22, 1963 [12]. Brenner extended Dougherty's work on the nutrition and development of $C$. elegans by establishing methods for genetic manipulations of the worm [4]. Such manipulations produce "mutants" that are amenable to behavioral and neuronal studies. Brenner went on to establish C. elegans as a model for understanding the biology of multi-cellular organisms. This was rationalized based on the intermediate position of $C$. elegans between bacteria and humans in regards to size and complexity. Subsequent work mapped the entire developmental cell lineage of $C$. elegans [39] as well as the organizational structure of its nervous system (the "connectome"), at cellular resolution [43]. It is now clear that 
the hermaphrodite worm possesses exactly 302 neurons in addition to approximately 6400 chemical synapses, 900 electrical synapses, and 1500 neuromuscular junctions [1].

Furthermore, C. elegans is the first multicellular organism for which humans have acquired the fully sequenced genome [40]. The worm is also the first organism whose nervous system humans have acquired a complete wiring diagram for. In addition to these monumental achievements, $C$. elegans has enjoyed many more "firsts" as a research organism, including: the identification of the first genes encoding axon guidance [17], the first multicellular organism to demonstrate the utility of Green Fluorescent Protein (GFP) as a biological marker of gene expression [5], the first organism for which humans have discovered the specific gene encoding the membrane receptor responsible for transducing olfactory signals in a chemosensitive neuron [36], the first use of optogenetics to evoke behavior via the light-sensitive Channelrhodopsin-2 ion channel in an intact animal [27], and the first discovery of a nematode virus [11]. At the time of writing, there are approximately 1000 laboratories in the world that are dedicated to C. elegans research. This number is monitored in an online database hosted by WormBase.org.

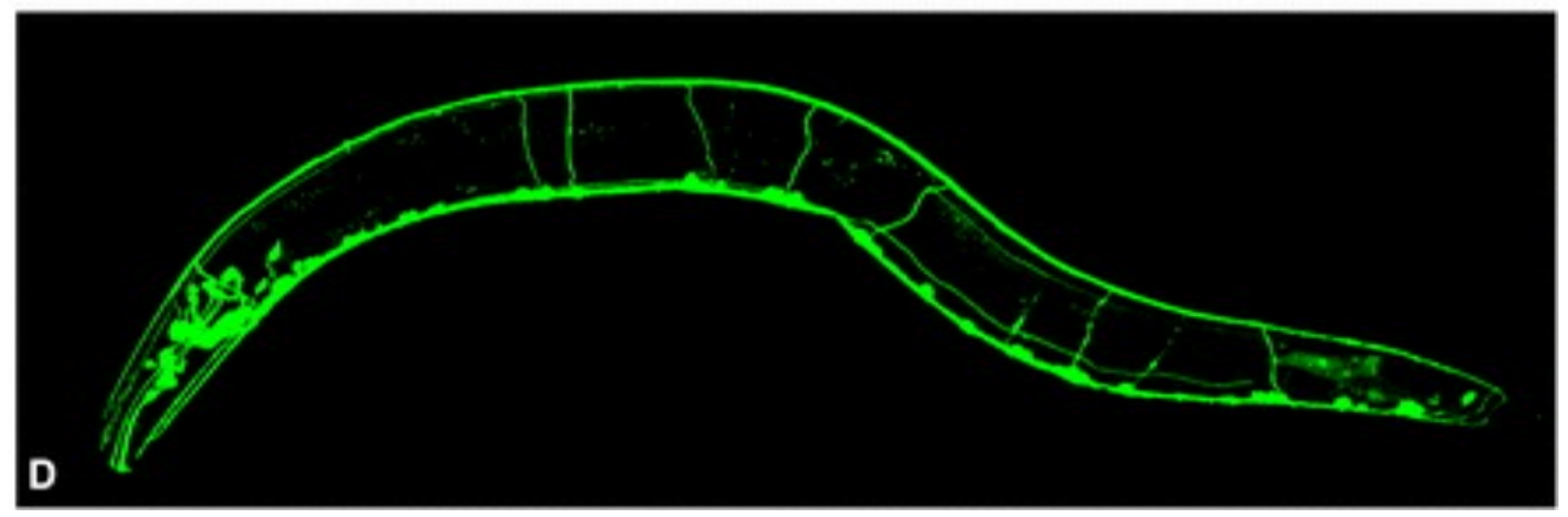

Figure 1. A microscope image of C. elegans with its nervous system labelled by a green fluorescent protein (GFP) reporter. Image from WormBook.org [7]. Copyright 2015 Oxford University Press. 
Many of the progressions made first with C. elegans have had wide applicability to other biological systems. For example, the use of both GFP and optogenetics is now commonplace in experimental biology [9] [37]. Rightly so, both of these methods comprise non-invasive techniques for making precise observations and manipulations of delicate biological cultures [33] or, in some cases, entire organisms (see figure 1) [14].

\section{Motivations}

The effect of temperature on physiology has long been a topic of scientific research [3] [16]. Temperature is not only a critical factor implicated in the dynamics of life at the organismal level [19], but also at the scales of ecosystems [46] and biospheres [35]. Given the fact that the large majority of modern-day humans rely on food that is cultivated or manufactured from a farm or factory, and that these agricultural industries succeed only under optimal physical and chemical conditions [31] [8] that are regulated by complex processes involving numerous biological players, a "global" parameter like temperature which affects all of these factors in distinct ways may be important to monitor and study. This is an especially urgent suggestion in the wider context of human-induced climate change, which has already been shown to disrupt entire economies [2].

\section{Subject of Study}

Many biological systems display a behavior known as "thermotaxis," which refers to changes in the motion of an organism based on the temperature of its surroundings. It is well-established that C. elegans exhibits thermotaxis to various extents across a range of temperatures surrounding its cultivation temperature (symbolized as " $T_{C}$ ", throughout). The cultivation temperature of a lab animal is the temperature at which that animal is allowed to feed and reproduce. Loosely-speaking, starved worms tend to navigate away from $T_{C}$ while satiated worms tend to navigate toward $T_{C}$. Thermotaxis appears to be connected to chemotaxis in C. elegans, since some genetically-modified (mutant) worms with a reduced thermotaxis ability also have a reduced ability to detect chemicals [18]. Importantly, there is a subtle asymmetry in the thermotaxis of C. elegans. When a satiated worm experiences temperatures 3 degrees above or below $T_{C}$, it will successfully migrate toward $T_{C}$ if placed in a sufficiently steep and uniform thermal gradient. However, if the experienced 
temperature of a satiated worm is 6 degrees above or below $T_{C}$, there is a difference between the cold worms and the hot worms. The worms held at 6 degrees below $T_{C}$ almost completely fail to migrate to warmer temperatures. On the other hand, worms held at 6 degrees above $T_{C}$ still migrate to cooler temperatures, albeit with less success than those held at only 3 degrees above $T_{C}$ $[20]$.

The anatomical sites where temperature signals are first processed by the worm have been identified as the amphid finger-like ciliated (AFD) neurons, which form a bilaterally-symmetric pair [26] In vivo studies show that the AFD neurons encode temperature changes with parallel changes in intracellular concentration of free calcium ions. This quantity will be succinctly referred to using the notation $\left[\mathrm{Ca}^{2+}\right]$, where "Ca" refers to the element "calcium", "2+" to the charge of the ion, and square brackets "[ ]" denoting "intracellular concentration of". Ambient temperatures that are above a threshold determined by $T_{C}$ result in a higher baseline $\left[\mathrm{Ca}^{2+}\right]$ level in the AFD neurons. Sinusoidal oscillations in ambient temperature with amplitudes as small as $0.05^{\circ} \mathrm{C}$ result in phase-locked $\left[\mathrm{Ca}^{2+}\right]$ oscillations in the AFD neurons [6] It has also been shown through in vivo electrophysiology studies that the expression of certain types of transmembrane proteins in AFD are necessary for the ability of these cells to process temperature signals via thermoreceptor currents thru ion channels embedded in the neuronal membrane [32] The mechanisms by which temperature fluctuations result in ionic currents and $\left[\mathrm{Ca}^{2+}\right]$ oscillations in the AFD neurons are not yet fully resolved. Here, computer simulations and mathematical analysis are used to study thermoreception in the AFD neurons of $C$. elegans, as encoded by the $\left[\mathrm{Ca}^{2+}\right]$ and membrane electrodynamics of this cell during various temperature stimuli.

A schematic diagram of the right AFD neuron (AFDR) is depicted in figure 2 and a schematic diagram of the left AFD neuron (AFDL) is depicted in figure 3. Both images have been taken from WormAtlas.org [47]; the image in figure 3. has been modified by the author. Connected by gap junction, there is no difference between the left and right AFD neurons in a healthy worm, other than their respective positions. Thus, they will be collectively referred to as "AFD," throughout. 


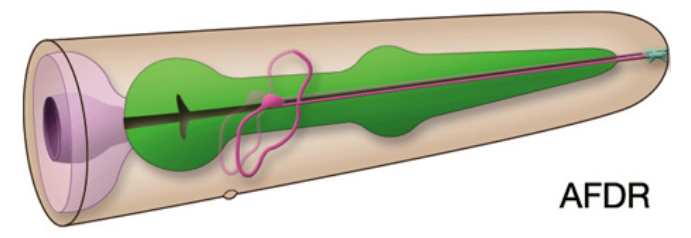

Figure 2. Schematic representation of the AFDR neuron within the head of $C$. elegans. Copyright 2002-2021 WormAtlas

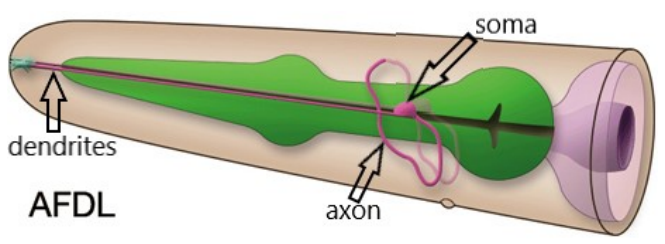

Figure 3. Schematic representation of the AFDL neuron within the head of $C$. elegans, with anatomical compartments labeled. Copyright 2002-2021 WormAtlas

The dendrites, soma, and axon are labeled in the AFDL neuron schematic of figure 3.. Of particular interest here are the dendrites, which terminate at the nose of the worm (on the right of figure 2 and on the left of figure 3) and are covered with finger-like sensory endings (see figure 4) that possess proteins necessary for thermosensation [28]. The green structure surrounding the magentacolored AFD neurons in figures 2 and 3 is a glial cell known as the amphid sheath. It completely envelops the AFD neuron's sensory endings and modifies their function and morhpology via the potassium-chloride $\left(\mathrm{K}^{+} / \mathrm{Cl}^{-}\right)$transporter, which pumps these ions across the AFD membrane [38]. The microvilli seen in figure 4 do not form if the amphid sheath is absent. Without the microvilli and surrounding amphid sheath, the $\left[\mathrm{Ca}^{2+}\right]$ responses of AFD to a temperature ramp are malformed and defective [41].

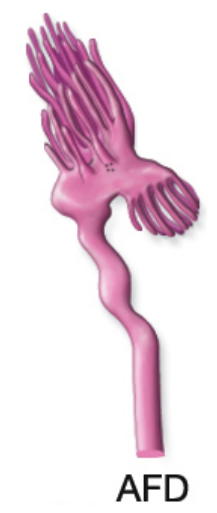

Figure 4. Illustration of the sensory endings of AFD. Copyright 2002-2021 WormAtlas 


\section{CHAPTER II: INTRACELLULAR CALCIUM DYNAMICS}

\section{Model Equations}

A mathematical model for the salt-sensitive $\left[\mathrm{Ca}^{2+}\right]$ response of the right amphid neuron with single ciliated endings (ASER) in C. elegans [25] has been modified with the intent to model the thermo-sensitive $\left[\mathrm{Ca}^{2+}\right]$ response of the AFD neurons. The dimensionless "Q10" factors $\rho=\rho(T)$ and $\phi=\phi(T)$ have been introduced, as is often done in neuron models [13], to account for the temperature $T$. These quantities are based on the Arrhenius law from chemistry, and give a measure of how the speed of a biochemical reaction changes when the ambient temperature $T$ changes by $10^{\circ} \mathrm{C}$. The two parameters $\rho$ and $\phi$ affect the speed of calcium changes in the model neuron here, without explicitly accounting for any specific chemical reaction(s). The model equations are as follows:

$$
\begin{aligned}
\frac{d c_{d}}{d t} & =\frac{\rho}{\tau_{d}}\left[-c_{d}+Y_{d} y_{d}+D\left(W_{d} c_{s}-c_{d}\right)\right]+I(t) \\
\frac{d c_{s}}{d t} & =\frac{\rho}{\tau_{s}}\left[-c_{s}+Y_{s} y_{s}+D\left(c_{d}+c_{a}-c_{s}\right)\right] \\
\frac{d c_{a}}{d t} & =\frac{\rho}{\tau_{a}}\left[-c_{a}+Y_{a} y_{a}+D\left(W_{a} c_{s}-c_{a}\right)\right] \\
\frac{d y_{i}}{d t} & =-\phi A c_{i}, \quad \text { if } I(t) \neq 0 \quad i=d, s, a \\
y_{i} & =0 \quad, \quad \text { if } I(t)=0 \\
\rho & =\rho(T)=\rho_{0}^{\left(T-T_{C}\right) / 10} \\
\phi & =\phi(T)=\phi_{0}^{\left(T-T_{C}\right) / 10}, a
\end{aligned}
$$

where $t$ is time in seconds. $c_{d}, c_{s}$, and $c_{a}$ are dimensionless quantities corresponding to the $\left[\mathrm{Ca}^{2+}\right]$ in the dendrites, soma, and axon of AFD, respectively. These concentrations are strongly correlated to the neuronal activity in each compartment, with time constants $\tau_{d}, \tau_{s}$, and $\tau_{a}$ (in seconds). $D$ is a dimensionless constant describing the rate of calcium diffusion between adjacent compartments. $I(t)$ is a time-dependent input to the cell. $Y_{i}(i=d, s, a)$ is the magnitude of the corresponding inactivation variable $y_{i}$. $W_{d}$ and $W_{a}$ are weights for calcium diffusion from the soma to the dendrite and from the soma to the axon, respectively. The values of these weights vary between 0 and 1 depending on the relative activity between dendrite and axon. For more details, see appendix A 
in chapter. The parameter $A$ represents the time scale of the inactivation $y_{i}$. Large values of $A$ produce faster inactivation, and vice-versa. $\rho$ represents the dependence of neuronal activation, in the form of $\left[\mathrm{Ca}^{2+}\right]$ increases, on temperature. $\phi$ represents the $\left[\mathrm{Ca}^{2+}\right]$ inactivation's temperature dependence. The temperature coefficients $\rho_{0}$ and $\phi_{0}$ control the magnitude of the temperature dependence of $c_{i}$ and $y_{i}$, respectively. $T$ is the ambient temperature and $T_{C}$ is the cultivation temperature of the worm (as first denoted in section ), both in ${ }^{\circ} \mathrm{C}$. All of the parameters and calcium concentrations are positive quantities. The inactivation variables $y_{i}$ take negative values and are non-zero only when a stimulus is present (i.e. when $I(t) \neq 0$ ). 
Single Response at Constant Temperature

It has been shown that the AFD neurons respond to temperature within a critical 20-second time window [41]. The response of the model AFD neurons' $\left[\mathrm{Ca}^{2+}\right]$ to a single 20 -second pulse of $I(t)=1$ for three different constant values of ambient temperature $T$ is shown in figures 5,6 , and 7 for the dendrites, soma, and axon, respectively.

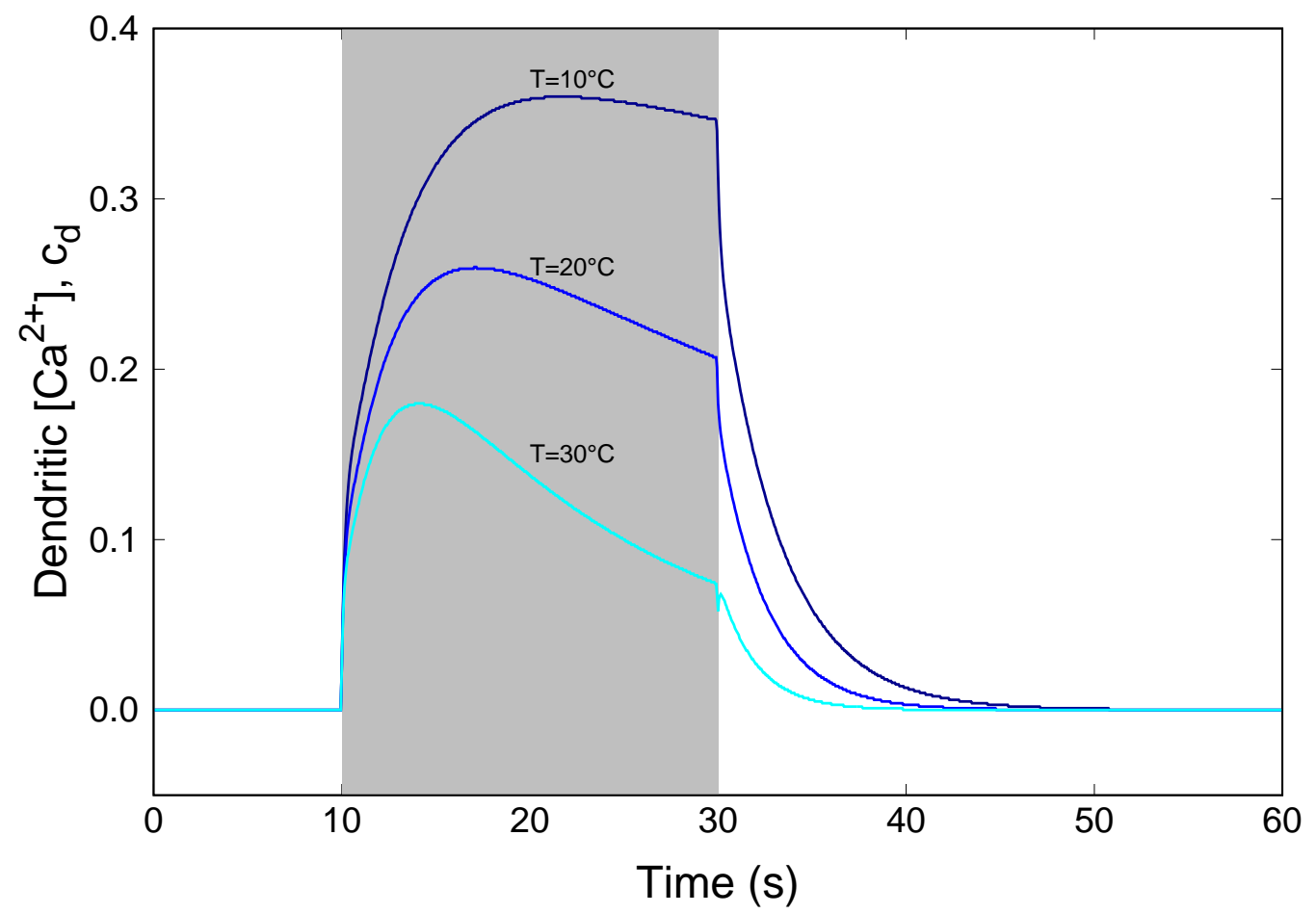

Figure 5. Dendritic calcium response of simulated AFD neuron to a 20-second stimulus for three different constant temperatures. The gray region represents the duration when $I(t)=1$. Outside of the gray region, $I(t)=0 . T_{C}=20^{\circ} \mathrm{C}, \rho_{0}=1.3, \phi_{0}=3.0$.

There is little difference in the qualitative shape of the $\left[\mathrm{Ca}^{2+}\right]$ time series across compartments. In all anatomical compartments, a $\left[\mathrm{Ca}^{2+}\right]$ response at higher temperatures peaks quicker and has a larger decay rate than a $\left[\mathrm{Ca}^{2+}\right]$ response at lower temperatures. The $\left[\mathrm{Ca}^{2+}\right]$ responses in the soma have the largest magnitude, followed by the dendrites, and then the axon. 


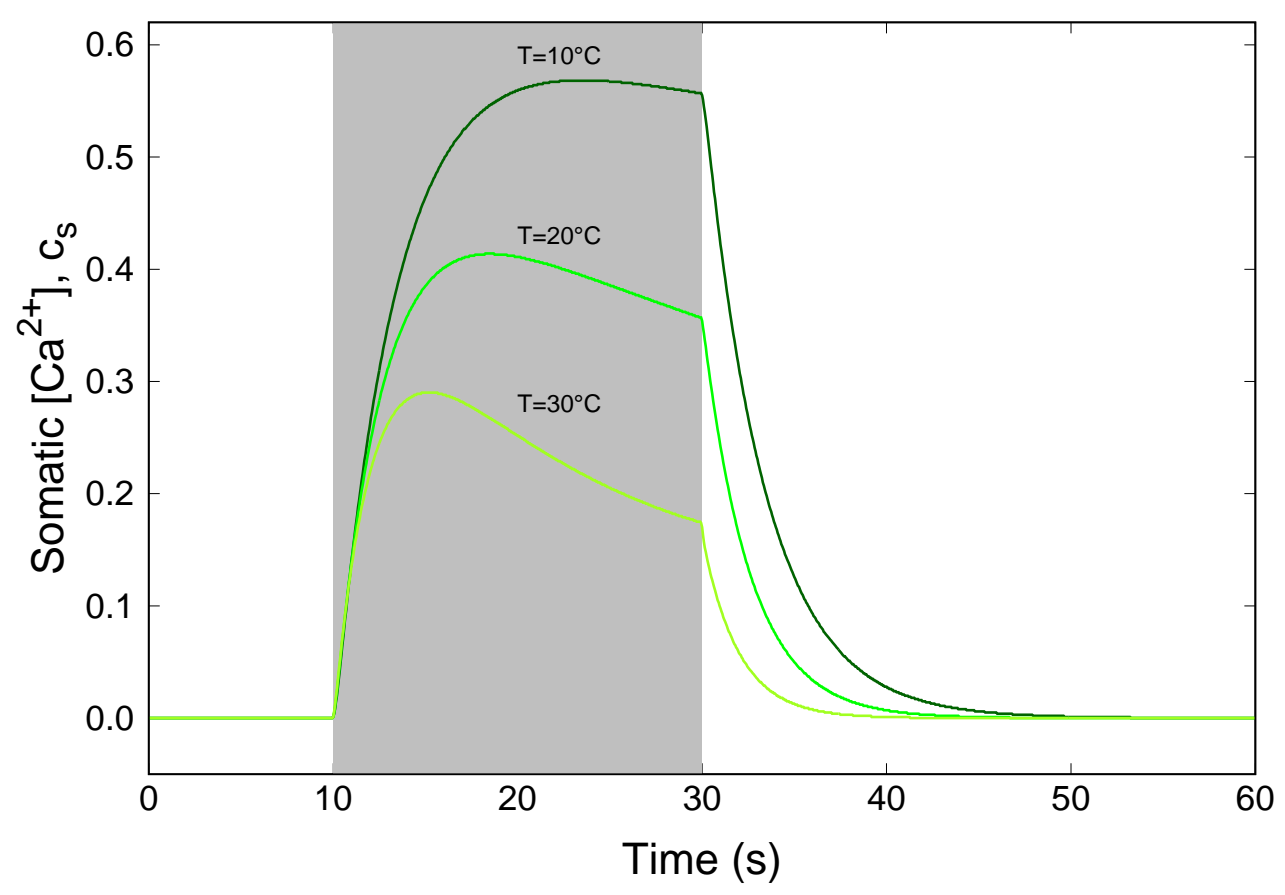

Figure 6. Somatic calcium response of simulated AFD neuron to a 20-second stimulus for three different constant temperatures. The gray region represents the duration when $I(t)=1$. Outside of the gray region, $I(t)=0 . T_{C}=20^{\circ} \mathrm{C}, \rho_{0}=1.3, \phi_{0}=3.0$.

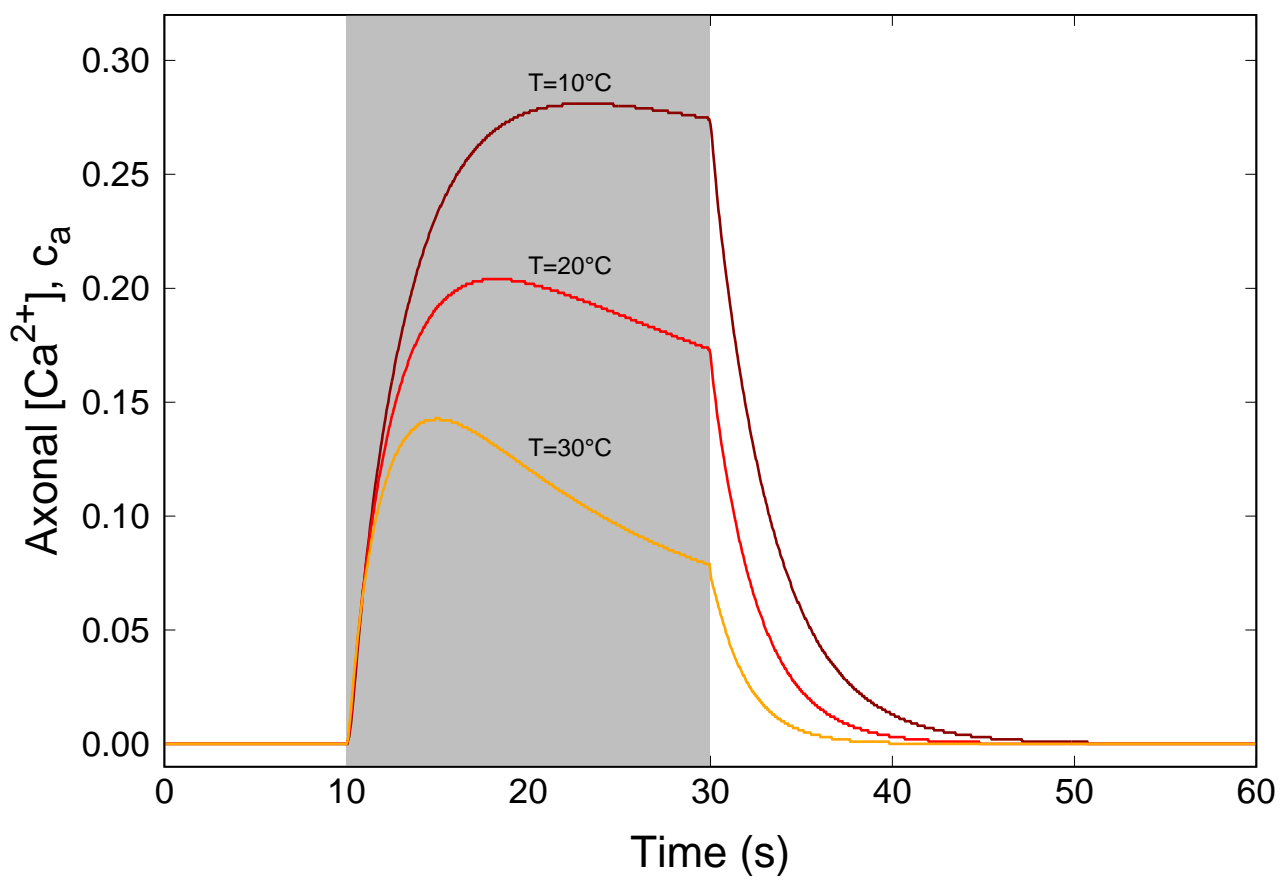

Figure 7. Axonal calcium response of simulated AFD neuron to a 20-second stimulus for three different constant temperatures. The gray region represents the duration when $I(t)=1$. Outside of the gray region, $I(t)=0 . T_{C}=20^{\circ} \mathrm{C}, \rho_{0}=1.3, \phi_{0}=3.0$. 
The stimulus duration is manually lengthened from 20 seconds to 60 seconds for the remainder of the plots in this subsection, purely to see what parameter values result in responses that do not fall within the critical 20-second time window. Figure 8 shows the normalized response of the model AFD neuron to a 60 -second $I(t)=1$ pulse for three different $\phi_{0}$ values when held $10^{\circ} \mathrm{C}$ above $T_{C}$. This result shows that larger $\phi_{0}$ results in a higher sensitivity to warm temperatures in the form of a larger decay rate of $c_{d} / c_{d \text { MAX }}$ during the $I(t)$ pulse. Across all $\phi_{0}$ values, the peak times (values of $t$ for which $c_{d} / c_{d \mathrm{MAX}}=1$ ) differ little in figure 8 .

Figure 9 shows the response of the model AFD neuron to a 60 -second $I(t)=1$ pulse for three different $\rho_{0}$ values when held $10^{\circ} \mathrm{C}$ above $T_{C}$. This result shows that larger $\rho_{0}$ results in a higher sensitivity to warm temperatures in the form of a quicker peak time during the $I(t)$ pulse. The decay rates of $c_{d} / c_{d \mathrm{MAX}}$ during the $I(t)=1$ pulse differ little across various values of $\rho_{0}$ in figure 9.

The trend observed in figures 8 and 9 is reversed in figures 10 and 11 , where $T<T_{C}$ instead of $T>T_{C}$. From figure 10, it can be seen that larger $\phi_{0}$ results in a slower decay rate during the pulse when $T<T_{C}$. The difference in peak times across $\phi_{0}$ values in figure 10 is, again, small. Figure 11 shows that that peak times differ substantially across $\rho_{0}$ values for dendrites held at temperatures $T<T_{C}$. The trace for $\rho_{0}=3$ in figure 11 just begins to start its decay around the 30 -second mark, which is the 20 -second mark with respect to the start of the $I(t)=1$ stimulus at $t=10 \mathrm{~s}$. This shows that large values of $\rho_{0}\left(>3\right.$ or so, when $\left.\phi_{0}=3.0\right)$ result in neurons that do not respond within the critical 20 -second time window at cold temperatures.

The trend of $\left[\mathrm{Ca}^{2+}\right]$ peak times in the dendrites as a function of $\phi_{0}$ and $\rho_{0}$ is seen for a wider range of Arhennius parameters in figures 12 and 13. In these figures, a 60-second pulse of $I(t)=1$ is exerted on the model AFD neuron for some values of $T, \phi_{0}$, and $\rho_{0}$, while the peak time with respect to the start of the 60-second pulse is recorded. It can be seen in figure 12 that dendrite peak time is approximately linear with respect to temperature, across all $\phi_{0}$ values considered. On the other hand, figure 13 shows that the dendrite peak time becomes more nonlinear in temperature as $\rho_{0}$ is increased. Furthermore, figure 13 shows that the AFD model neurons with $\rho_{0}=3,4,5$ fall outside of the critical 20 -second time window when held at low temperatures. 


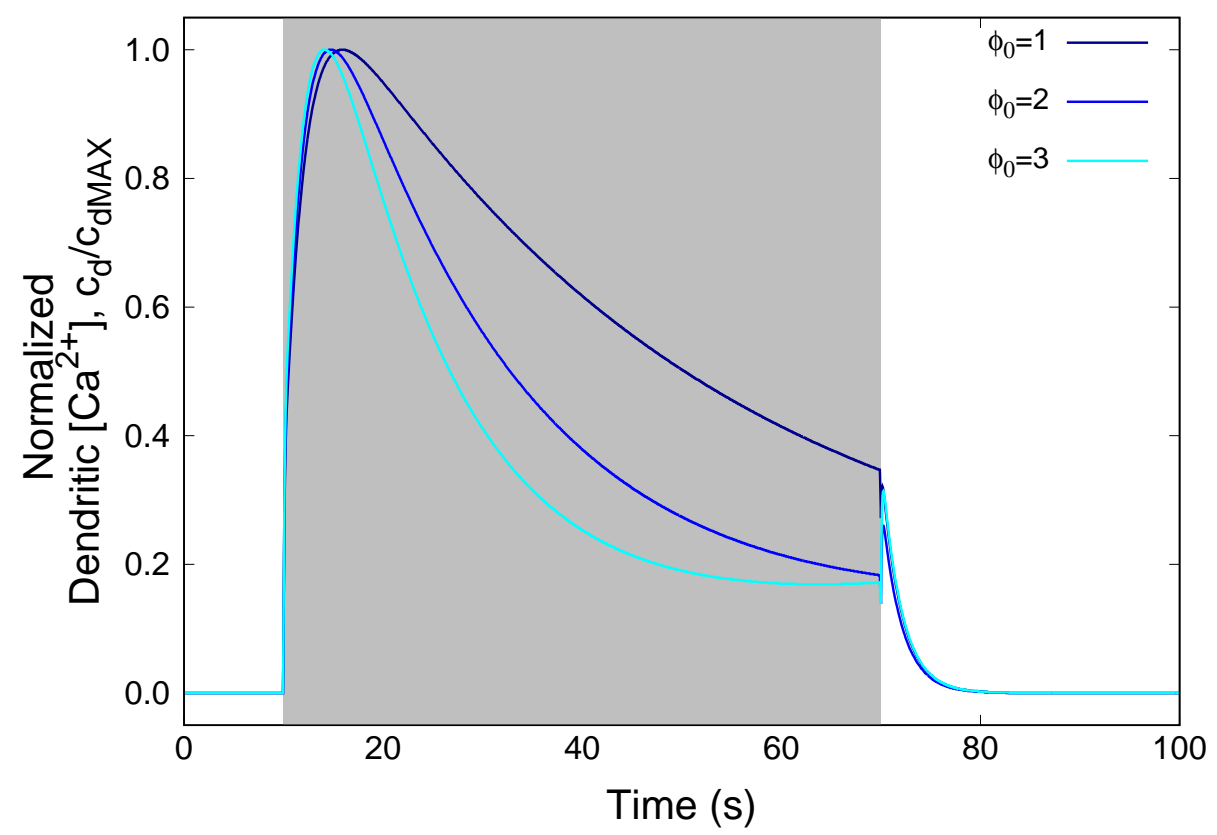

Figure 8. Normalized dendritic calcium response of simulated AFD neuron to a 60 -second stimulus for three different $\phi_{0}$ values, in warm conditions. The gray region represents the duration when $I(t)=1$. Outside of the gray region, $I(t)=0 . T=30^{\circ} \mathrm{C}, T_{C}=20^{\circ} \mathrm{C}, \rho_{0}=1.3$.

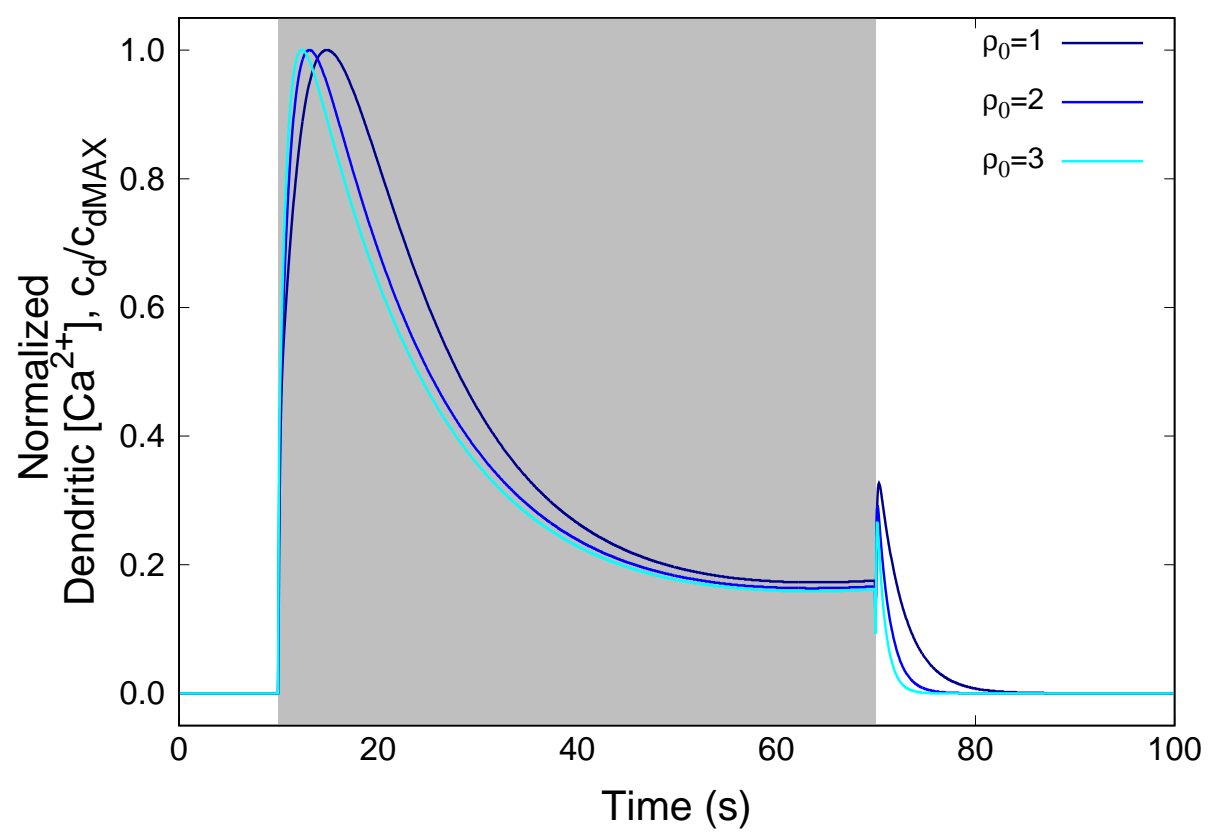

Figure 9. Normalized dendritic calcium response of simulated AFD neuron to a 60 -second stimulus for three different $\rho_{0}$ values, in warm conditions. The gray region represents the duration when $I(t)=1$. Outside of the gray region, $I(t)=0 . T=30^{\circ} \mathrm{C}, T_{C}=20^{\circ} \mathrm{C}, \phi_{0}=3.0$. 


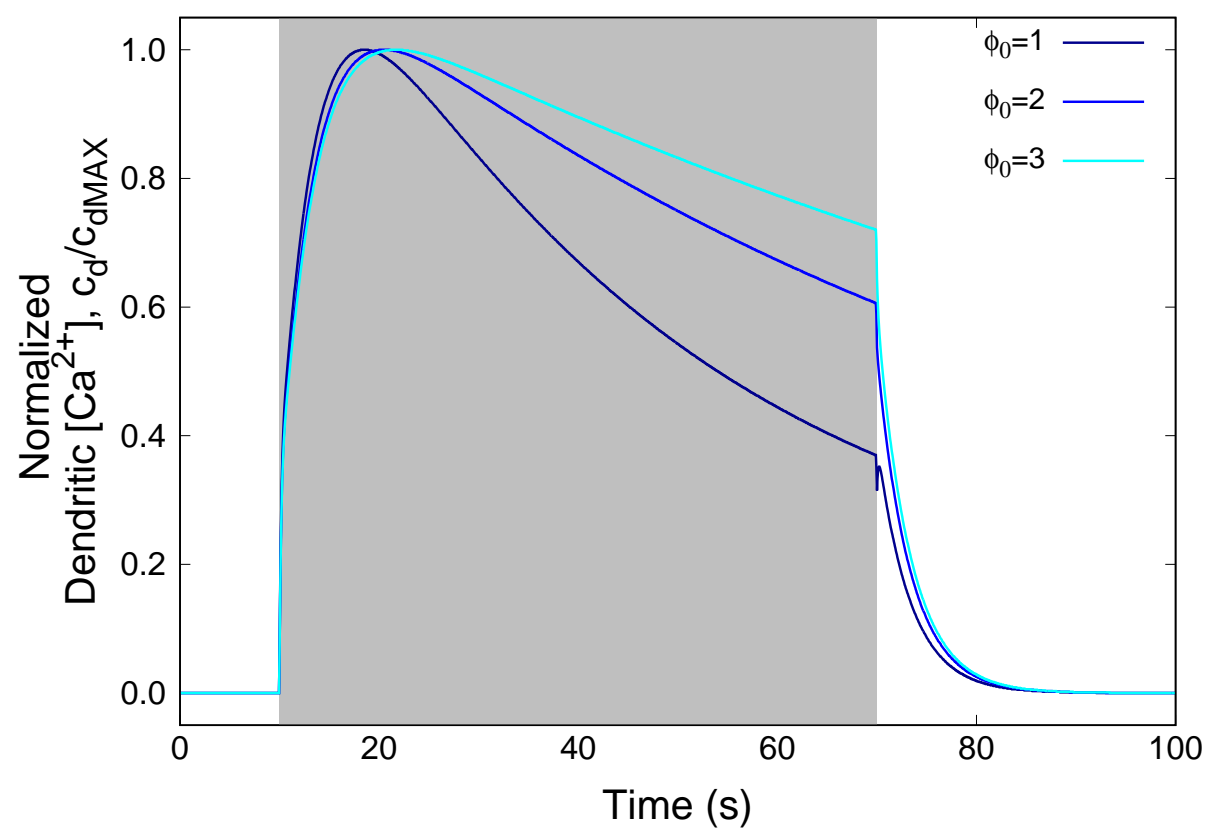

Figure 10. Dendritic calcium response of simulated AFD neuron to a 60-second stimulus for three different $\phi_{0}$ values, in cold conditions. The gray region represents the duration when $I(t)=1$. Outside of the gray region, $I(t)=0 . T=10^{\circ} \mathrm{C}, T_{C}=20^{\circ} \mathrm{C}, \rho_{0}=1.3$.

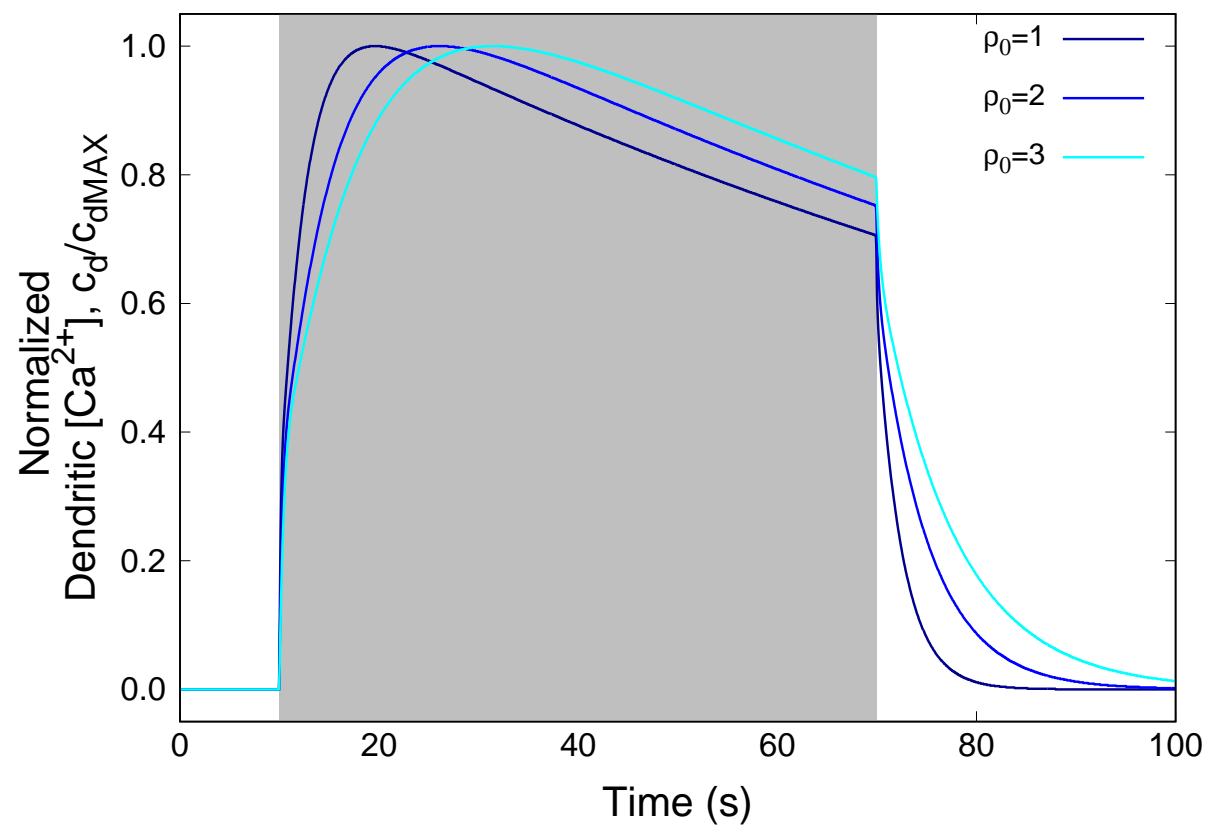

Figure 11. Dendritic calcium response of simulated AFD neuron to a 60-second stimulus for three different $\rho_{0}$ values, in cold conditions. The gray region represents the duration when $I(t)=1$. Outside of the gray region, $I(t)=0 . T=10^{\circ} \mathrm{C}, T_{C}=20^{\circ} \mathrm{C}, \phi_{0}=3.0$. 


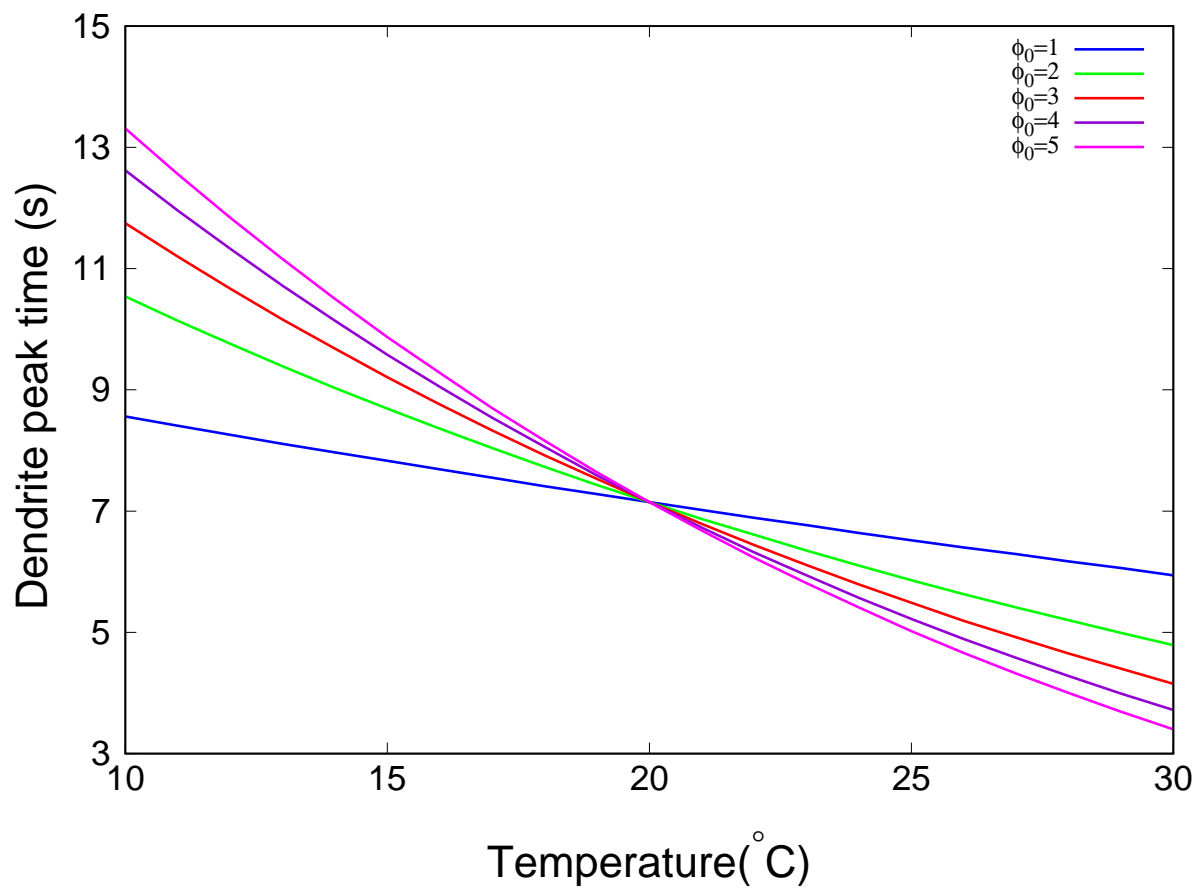

Figure 12. Peak time of $c_{d}$ during a 60-second pulse of $I(t)=1$ for various $\phi_{0}$ and $T$, as measured from the start of the stimulus. $T_{C}=20^{\circ} \mathrm{C}, \rho_{0}=1.3$.

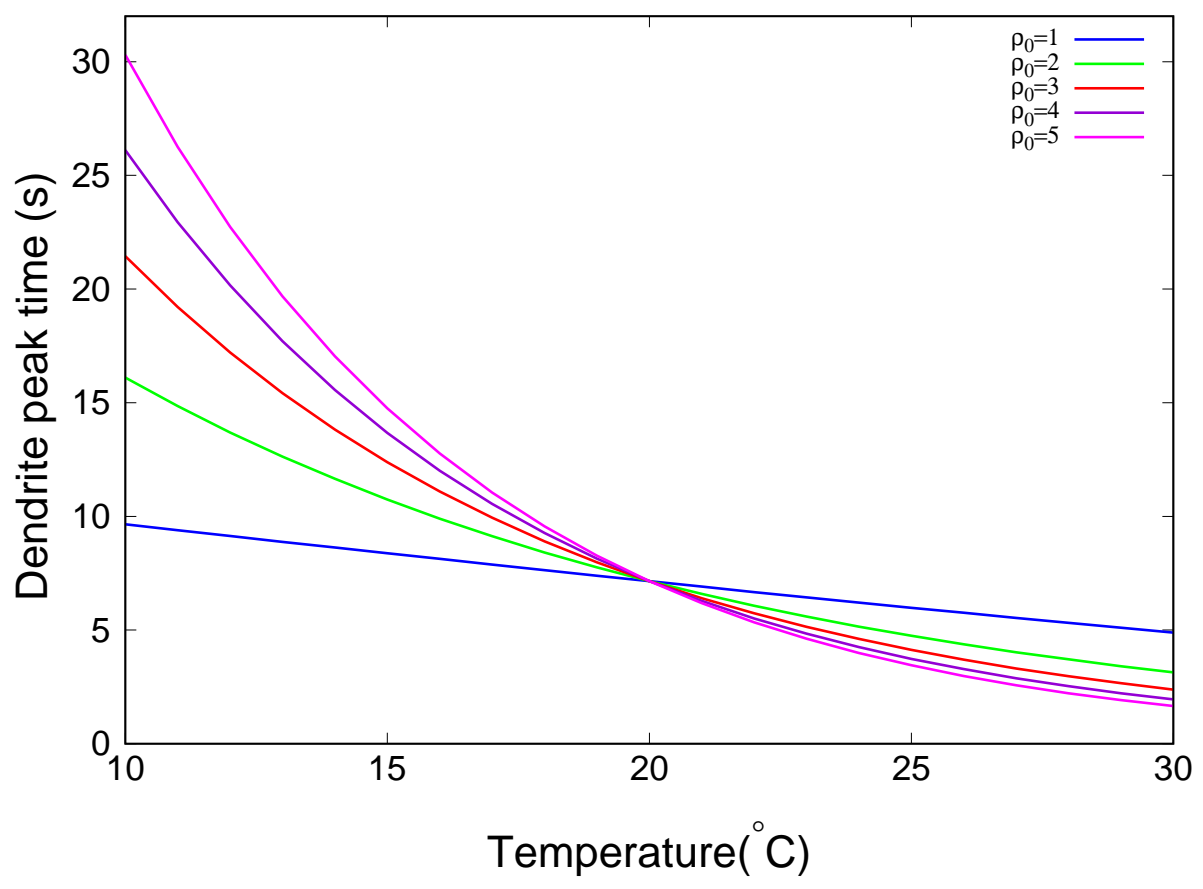

Figure 13. Peak time of $c_{d}$ during a 60-second pulse of $I(t)=1$ for various $\rho_{0}$ and $T$, as measured from the start of the stimulus. $T_{C}=20^{\circ} \mathrm{C}, \phi_{0}=3.0$. 


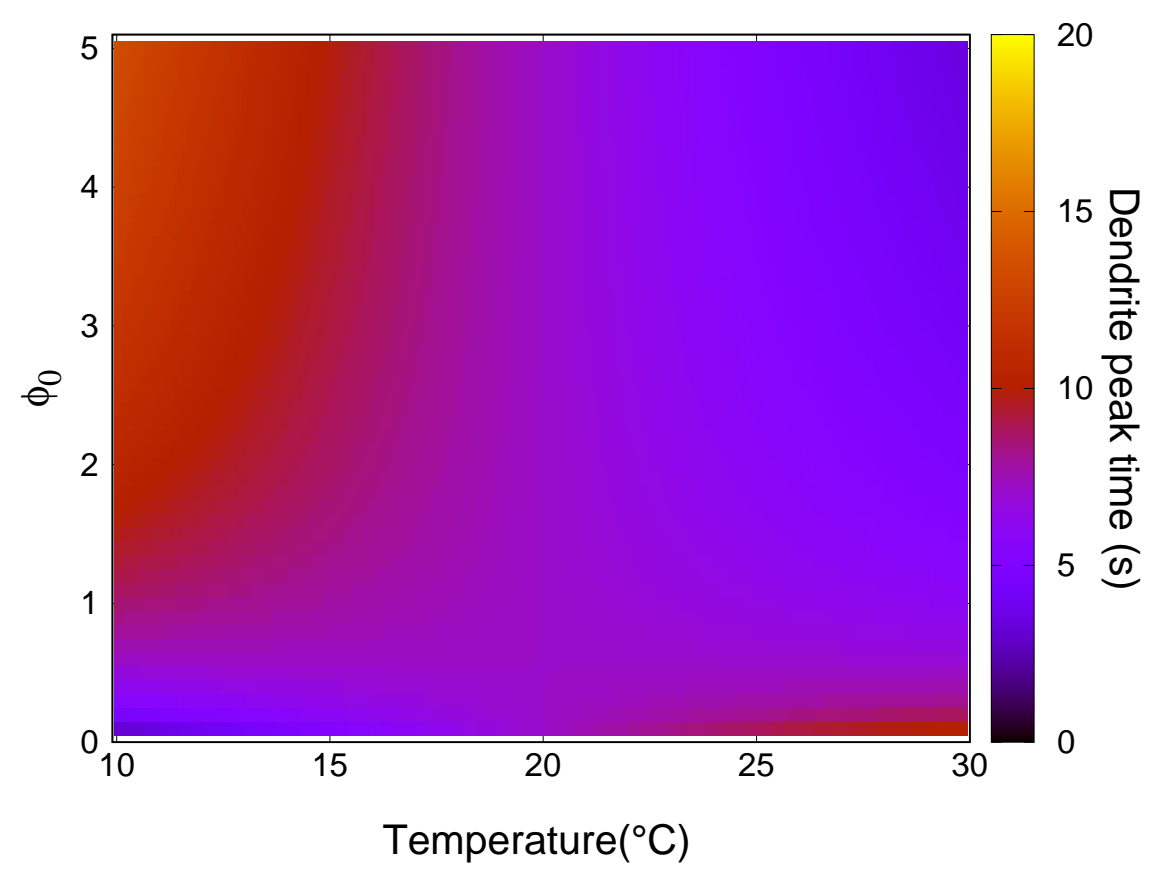

Figure 14. Peak time of $c_{d}$ during a 60 -second pulse of $I(t)=1$ versus $T$ and $\phi_{0} . T_{C}=20^{\circ} \mathrm{C}$, $\rho_{0}=1.3$.

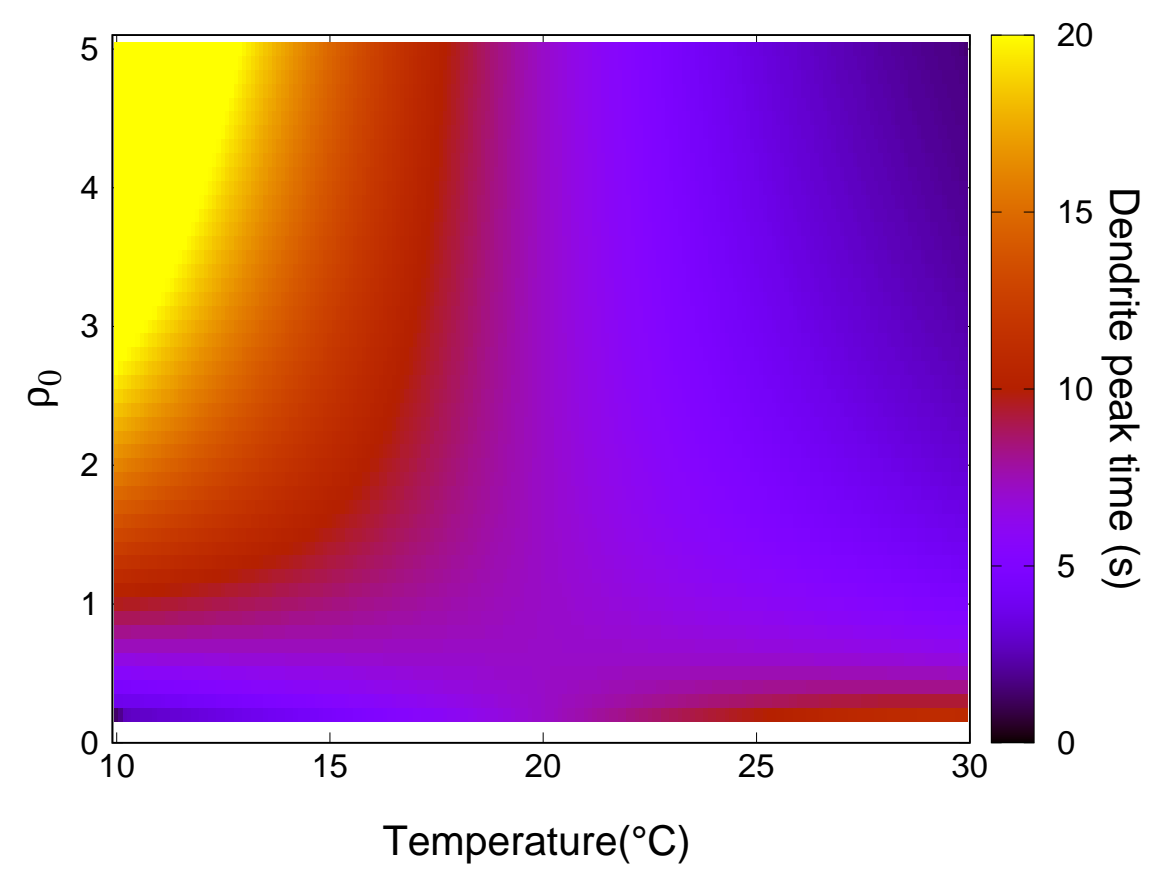

Figure 15. Peak time of $c_{d}$ during a 60 -second pulse of $I(t)=1$ versus $T$ and $\rho_{0}$. Any peak time $\geq 20 \mathrm{~s}$ appears as yellow. $T_{C}=20^{\circ} \mathrm{C}, \phi_{0}=3.0$. 
Figure 12 shows that the dendrite peak time is a decreasing function of temperature whose slope increases as $\phi_{0}$ increases. The reason that all curves intersect at $T=20^{\circ} \mathrm{C}$ is because all models become equivalent when $\rho=\rho_{0}^{(20-20) / 10}=\phi=\phi_{0}^{(20-20) / 10}=1$ at $T=20^{\circ} \mathrm{C}$. Figure 13 shows that the dendrite peak time is a decreasing function of temperature when $\phi_{0}=3.0$ whose steepness and nonlinearity increase as $\rho_{0}$ increases. The curves with $\rho=3$ (red), $\rho_{0}=4$ (violet), and $\rho_{0}=5$ (magenta) in figure 13 all give a dendrite peak time greater than 20 seconds at a temperature of $10^{\circ} \mathrm{C}$. This pattern of $\rho$ and $\phi$ modulating the temperature sensitivity of the calcium response is seen for a continuum of $\phi_{0}$ and $\rho_{0}$ values in the color maps of figures 14 and 15, respectively. The range of the color (representing the dendrite peak time) has been manually set at $[0: 20]$ for both plots, so that it may be easy to see how these model neurons relate to the critical 20 -second time window of thermoreception by the AFD neurons. There is less variation in color for various $\phi_{0}$ in figure 14 when compared to the color variation for various $\rho_{0}$ shown in figure 15 , indicating that $\rho_{0}$ has a larger effect on the peak time of the calcium response. The top left yellow corner of figure 15 gives parameter values of $\rho_{0}$ and $T$ for which the calcium response takes longer than 20 seconds. 
Matching the Experimental Data

There have been multiple experimental works published where calcium imaging was performed in AFD while temperature was manipulated. The results in this section are of model simulations performed with the intent to match these experimental data. Figure 16 shows a simulation result that is qualitatively similar to the experimental data acquired by Clark et al. (2006) [6], which is reprinted for comparison here in figure 17. Figure 18 shows a simulation result that is qualitatively similar to figure 19, which also shows experimental data from Clark et al. (2006) [6]. Lastly, figure 20 shows a simulation result that is qualitatively similar to figure 21 , which shows experimental data reprinted from Yoshida et al. (2016) [45].

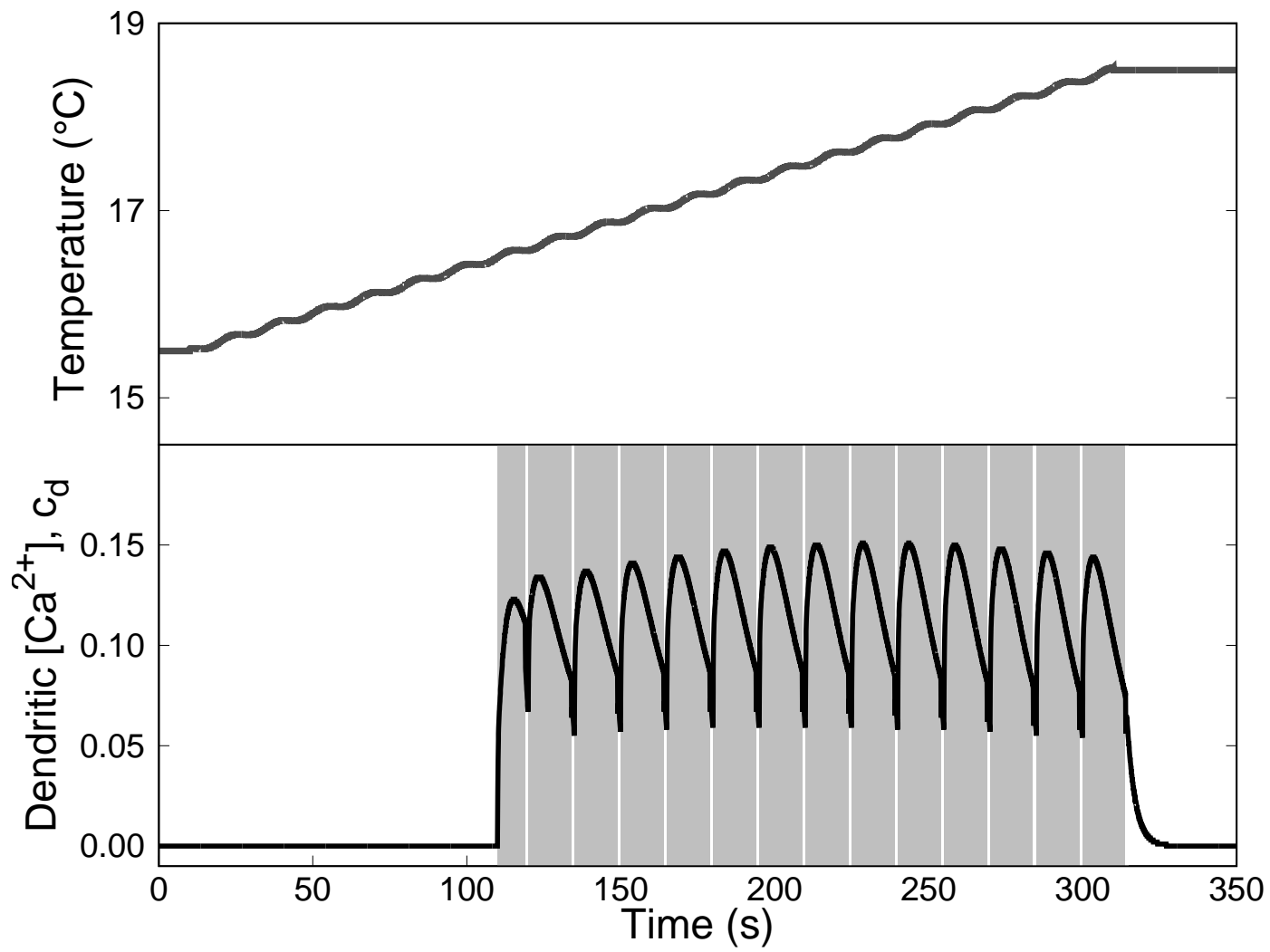

Figure 16. Dendritic calcium response of simulated AFD neuron to a linear ramping temperature with a sinusoidal component. The gray regions represent the durations when $I(t) \neq 0$. Outside of the gray regions, $I(t)=0 . T_{C}=20^{\circ} \mathrm{C}, \phi_{0}=3.0, \rho_{0}=1.3$. Qualitative match to experimental data in figure 17. 


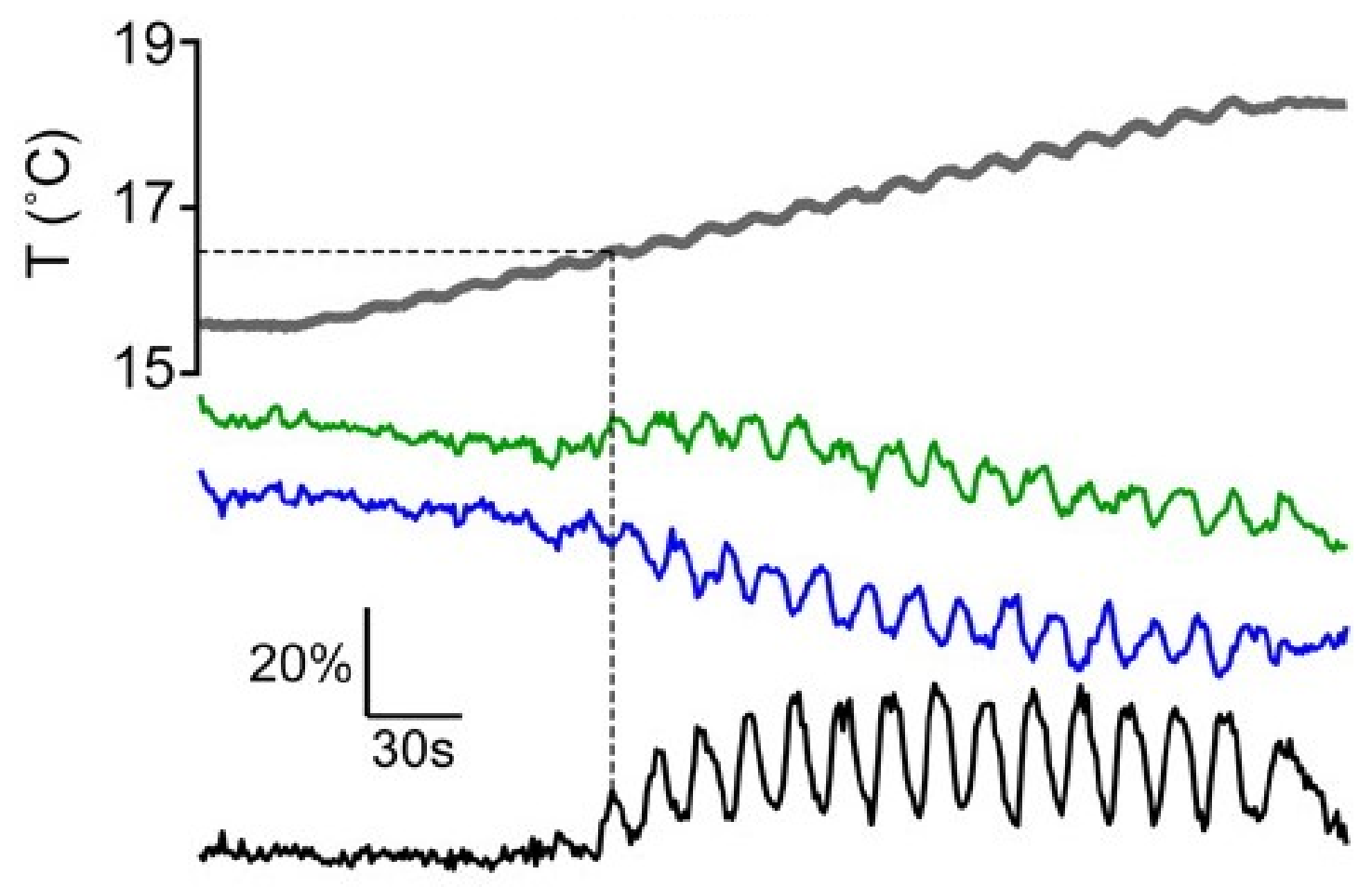

Figure 17. Experimental data from figure 1B of Clark et al. (2006) [6]: oscillating temperature ramp below $T_{C}=20^{\circ} \mathrm{C}$. The green trace depicts time-dependent emission of light by the cameleon calcium sensor yellow fluorescent protein (YFP) expressed in the AFD soma for this experiment. The blue trace shows emission by the cyan fluorescent protein (CFP) expressed in the AFD soma. The ratio of these two quantities is given by the black trace, which is a measure of the $\mathrm{Ca}^{2+}$ concentration in the AFD soma. These changes in $\mathrm{Ca}^{2+}$ are a result of the ambient temperature (shown in gray) exposed to the worm during this experiment. Copyright 2006 Society for Neuroscience. 


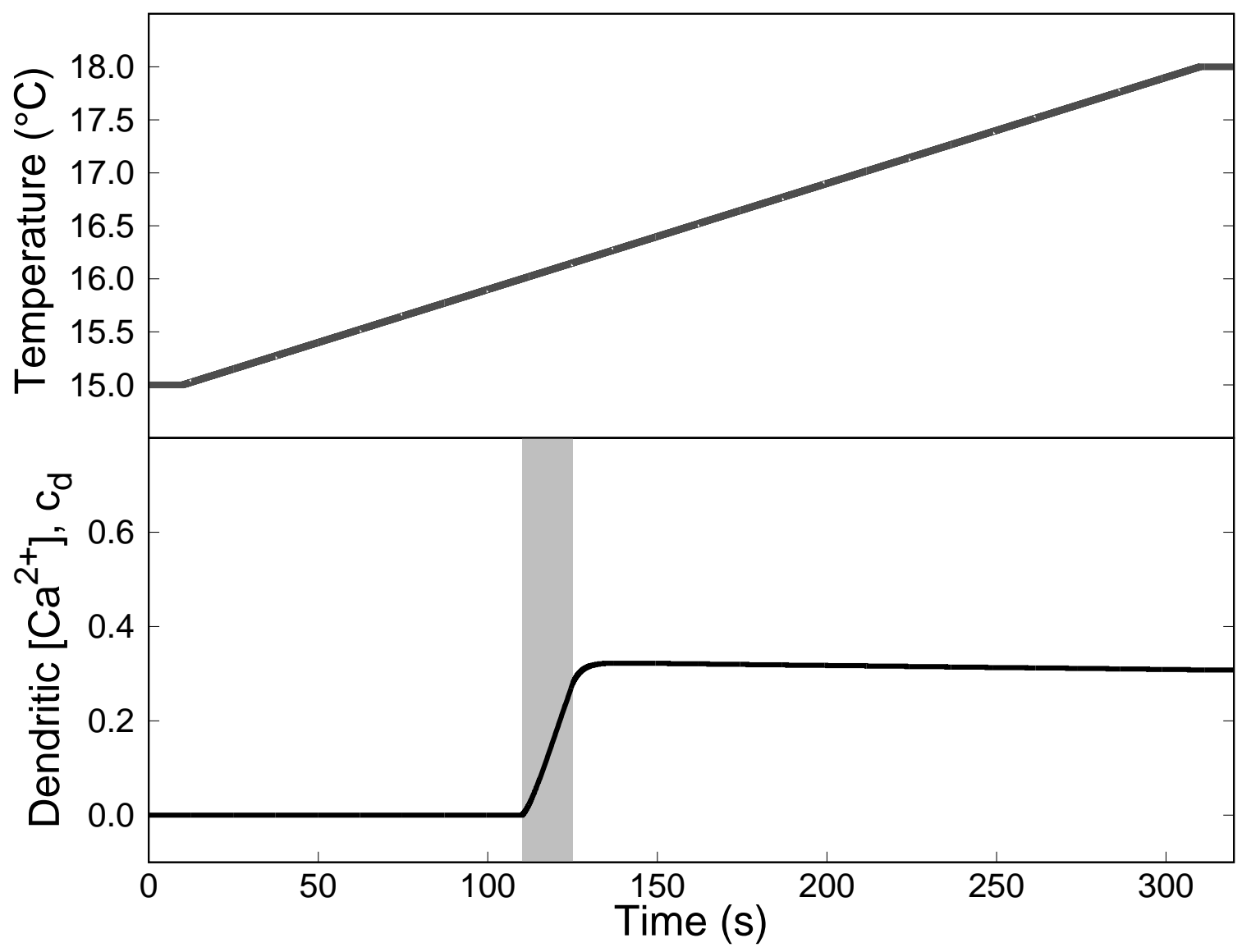

Figure 18. Dendritic calcium response of simulated AFD neuron to a linear ramping temperature below $T_{C}$. The gray region represents the duration when $\frac{d I}{d t} \neq 0$. Outside of the gray regions, $\frac{d I}{d t}=0 . T_{C}=20^{\circ} \mathrm{C}, \phi_{0}=3.0, \rho_{0}=1.3$. Qualitative match to experimental data in figure 19.

The equations underlying figure 18 above are a slight alteration from equations 1-7. Instead of setting $y_{i}=0$ when $I(t)=0$, the implementation in figure 18. has $\frac{d y_{i}}{d t}=0$ whenever $\frac{d I}{d t}=0$. Otherwise, $\frac{d y_{i}}{d t}=-\phi A c_{i}$ when $\frac{d I}{d t} \neq 0$. Additionally, the $I(t)$ time-dependence in figure 18 is coupled to temperature and time in the following way:

$$
I(t)= \begin{cases}0, & T<T_{C}-4 \\ \frac{t}{15}-\frac{110}{15}, & T \geq T_{C}-4 \text { AND } t<125 \mathrm{~s} \\ 1, & \text { otherwise }\end{cases}
$$




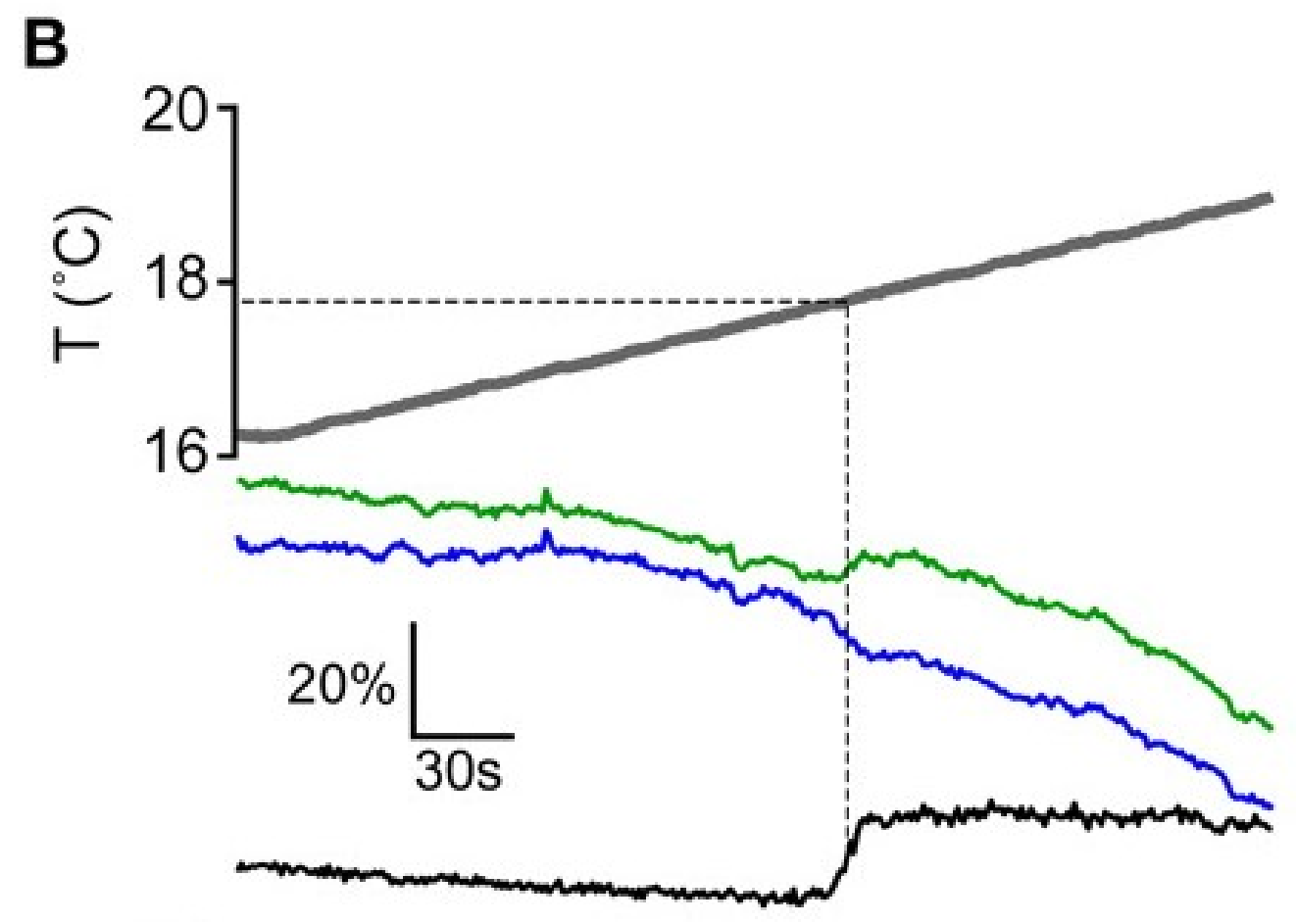

Figure 19. Experimental data from figure 1B of Clark et al. (2006) [6]: linear temperature ramp below $T_{C}=20^{\circ} \mathrm{C}$. The colors of the traces denote the same quantities as those colors do in figure 17 above. Copyright 2006 Society for Neuroscience. 


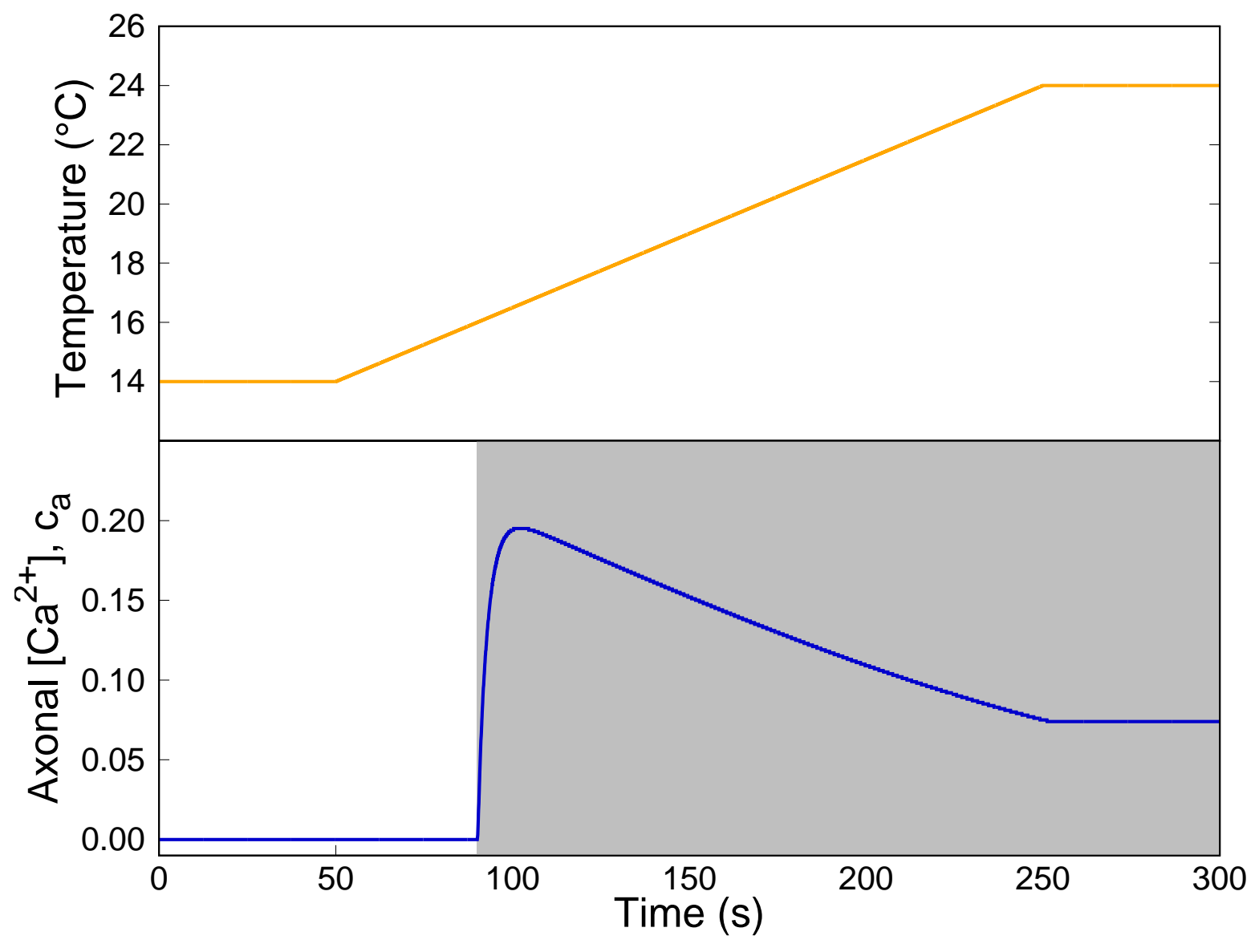

Figure 20. Axonal calcium response of simulated AFD neuron to a linear ramping temperature above $T_{C}$. The gray region represents the duration when $I(t)=0.8$. Outside of the gray region, $I(t)=0 . T_{C}=20^{\circ} \mathrm{C}, \phi_{0}=3.0, \rho_{0}=1.3$. Qualitative match to experimental data in figure 21.

The equations underlying figure 20 above are a slight alteration from equations 1-7. Instead of forcing $y_{i}=0$ when $I(t)=0$, the implementation in figure 20 has $\frac{d y_{i}}{d t}=0$ whenever $\frac{d T}{d t}=0$. Otherwise, $\frac{d y_{i}}{d t}=-\phi A c_{i}$ when $\frac{d T}{d t} \neq 0$. Additionally, the $I(t)$ time-dependence in figure 20 is coupled to temperature in the following way:

$$
I(t)= \begin{cases}0, & T \leq T_{C}-4 \\ 0.8, & T>T_{C}-4\end{cases}
$$



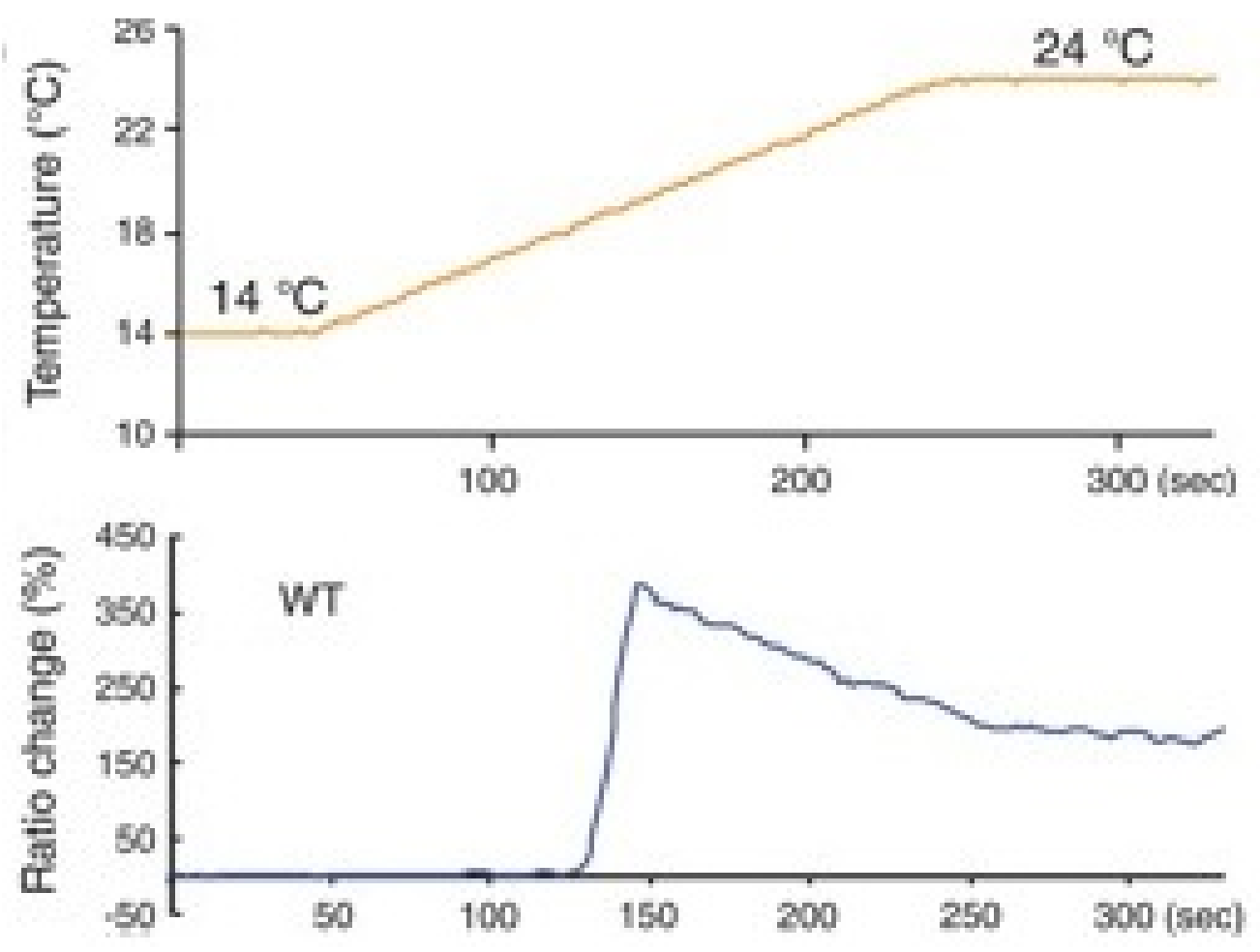

Figure 21. Experimental data from figure 1B of Yoshida et al. (2016) [45], reprinted with permission: linear temperature ramp above $T_{C}$. The top orange trace shows the ambient temperature exposed to the AFD neuron during this experiment. The bottom gray blue trace shows the ratio change of GCaMP3/RFP, which is a metric that scales positively with $\left[\mathrm{Ca}^{2+}\right]$. The worm from which these data are taken was cultivated at $17^{\circ} \mathrm{C}$. Copyright 2016 John Wiley \& Sons Inc.

Summary of the $\mathrm{Ca}^{2+}$ Model Results

The calcium model predicts that temperature affects a $\left[\mathrm{Ca}^{2+}\right]$ response in AFD by modulating its rate of decay and the timing of its peak. At temperatures $T>T_{C}$, increasing $\phi_{0}$ results in a steeper decay of the $\left[\mathrm{Ca}^{2+}\right]$ wave but changes the peak time negligibly. Similarly, increasing $\rho_{0}$ at temperatures $T>T_{C}$ shortens the peak time of the $\left[\mathrm{Ca}^{2+}\right]$ wave but does little to its rate of decay. Both of these trends are reversed when $T<T_{C}$. This can be seen for a wide range of parameter values in the color maps of figures 14 and 15. Besides the addition of $\rho$ and $\phi$, further modifications of the inactivations' dependence on the nature of the stimulus (in the form of $I(t)$ or temperature $T$ ) were made for the model to fit the data. 


\section{CHAPTER III: MEMBRANE ELECTRODYNAMICS}

This chapter explores a biophysical model of the ionic currents passing through the neuronal membrane of AFD during various temperature and voltage-clamp conditions. Such currents play an active role in altering the membrane voltage of the neuron, which is a primary quantity of interest. These currents are also involved in modulating the internal state of the AFD neurons, and affect intracellular $\left[\mathrm{Ca}^{2+}\right]$ signaling.

Model Equations with Constant Voltage Solution

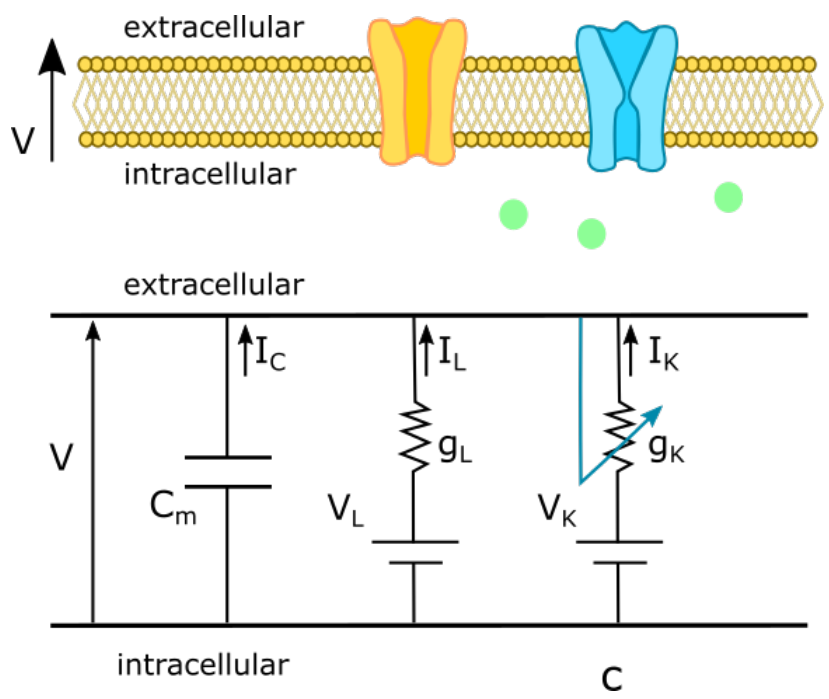

Figure 22. Circuit model of the AFD neuronal membrane. Schematic of phospholipid bilayer membrane of the AFD neuron with ion channels (top) and its electronic circuit description (bottom) [34].

Consider a neuron described by the electronic circuit diagram of figure 22 and corresponding equations:

$$
\begin{aligned}
C_{m} \frac{d V}{d t} & =I_{\mathrm{app}}-g_{K} m\left(V-V_{K}\right)-g_{L}\left(V-V_{L}\right) \\
\frac{d m}{d t} & =-\rho m+c \\
\frac{d c}{d t} & =-\rho c
\end{aligned}
$$


where $t$ is time in milliseconds (ms), $C_{m}$ is the membrane capacitance in picoFarads $(\mathrm{pF}), V$ is the membrane voltage in milliVolts $(\mathrm{mV}), I_{\text {app }}$ is the applied current in picoAmperes $(\mathrm{pA}), g_{K}$ is a constant scaling up the potassium ion $\left(\mathrm{K}^{+}\right)$conductance in nanoSiemens $(\mathrm{nS}), V_{K}$ is the Nernst potential for $\mathrm{K}^{+}$in $\mathrm{mV}, g_{L}$ is the maximum leak conductance in $\mathrm{nS}, V_{L}$ is the Nernst potential for the leak channels in $\mathrm{mV}, m$ is the dimensionless $\mathrm{K}^{+}$current activation, $\rho>0$ is a temperaturedependent parameter with units of $\mathrm{ms}^{-1}$, and $c>0$ is a variable with units $\mathrm{ms}^{-1}$ that correlates with the concentration of some intracellular chemical whose presence increases the activation of the $\mathrm{K}^{+}$channels. In figure $22, I_{C}=C_{m} \frac{d V}{d t}$ is the capacitive current, $I_{K}=g_{K} m\left(V-V_{K}\right)$ is the $\mathrm{K}^{+}$ current, and $I_{L}=g_{L}\left(V-V_{L}\right)$ is the leak current. The green circles represent the chemical $c$.

During a voltage clamp, we have $\frac{d V}{d t}=0$ and subsequently,

$$
I_{\text {app }}=g_{K} m\left(V-V_{K}\right)+g_{L}\left(V-V_{L}\right)
$$

from equation 10 above. The remaining two differential equations 11 and 12 can be solved analytically. Starting with equation 12: it is separable and can be solved by direct integration:

$$
\begin{aligned}
& \frac{d c}{d t}=-\rho c \\
& \frac{d c}{c}=-\rho d t
\end{aligned}
$$

Integrating both sides and isolating $c=c(t)$ yields

$$
c(t)=c_{0} e^{-\rho t}
$$

This is an exponential decay of $c$ from its initial value $c(0)=c_{0}>0$, where larger $\rho$ results in a quicker decay. This is consistent with $\rho=\rho(T)$ being an increasing function of temperature, since chemicals tend to diffuse away quicker at higher temperatures. Inserting the solution for $c$ into equation 11 yields

$$
\frac{d m}{d t}=-\rho m+c_{0} e^{-\rho t}
$$

which may be solved by seeking a function $m=m(t)$ of the form:

$$
m(t)=m_{H}(t) u(t)
$$


for some function $u(t)$ (to be determined), and where $m_{H}$ is given by the solution to the homogeneous equation:

$$
\dot{m}_{H}=-\rho m_{H}
$$

By the same argument that was used for finding $c(t)$, we have that

$$
m_{H}(t)=e^{-\rho t}
$$

where the integration constant $m_{H}(0)$ has been set equal to 1 . This may be done, without loss of generality, since any integration constant associated with $m_{H}$ may be embedded in the integration constant associated with $u(t)$. Taking the time derivative of equation 15 yields

$$
\frac{d m}{d t}=\frac{d m_{H}}{d t} u+m_{H} \frac{d u}{d t}
$$

Substituting in $\frac{d m_{H}}{d t}=-\rho m_{H}, m_{H}=e^{-\rho t}$ and $u=\frac{m}{m_{H}}$ to the above equation results in

$$
\frac{d m}{d t}=-\rho m+e^{-\rho t} \frac{d u}{d t}
$$

Adding $\rho m$ to both sides results in

$$
\frac{d m}{d t}+\rho m=e^{-\rho t} \frac{d u}{d t}
$$

From equation 14 , we also have that $\frac{d m}{d t}+\rho m=c_{0} e^{-\rho t}$. Inserting this into the left side of the equation directly above results in

$$
\begin{aligned}
c_{0} e^{-\rho t} & =e^{-\rho t} \frac{d u}{d t} \\
\frac{d u}{d t} & =c_{0}
\end{aligned}
$$

The solution for $u(t)$ is thus given by

$$
u(t)=c_{0} t+m_{0}
$$

where $u(0)=m(0)=m_{0}$. Therefore, the solution to the following dynamical system:

$$
\begin{aligned}
\frac{d m}{d t} & =-\rho m+c \\
\frac{d c}{d t} & =-\rho c
\end{aligned}
$$


is given by:

$$
\begin{aligned}
m(t) & =e^{-\rho t}\left(c_{0} t+m_{0}\right) \\
c(t) & =c_{0} e^{-\rho t}
\end{aligned}
$$

Equation 19 represents a simple exponential decay of $c$ from its intial value $c_{0}$ whose decay rate increases with increasing $\rho$. The function in equation 18, however, can display more interesting behavior. It is the different curves obtainable by this function that can account for the membrane current response of the AFD neuron during a voltage clamp. Various parameter values are explored in the following section to demonstrate the distinct regimes this function can display. 
Properties of the Potassium Activation during a Voltage Clamp

Letting $m_{0}=0$ and $c_{0}=4$ results in the function $m(t)=4 t e^{-\rho t}$, which is plotted for various values of $\rho$ in figure 23 below.

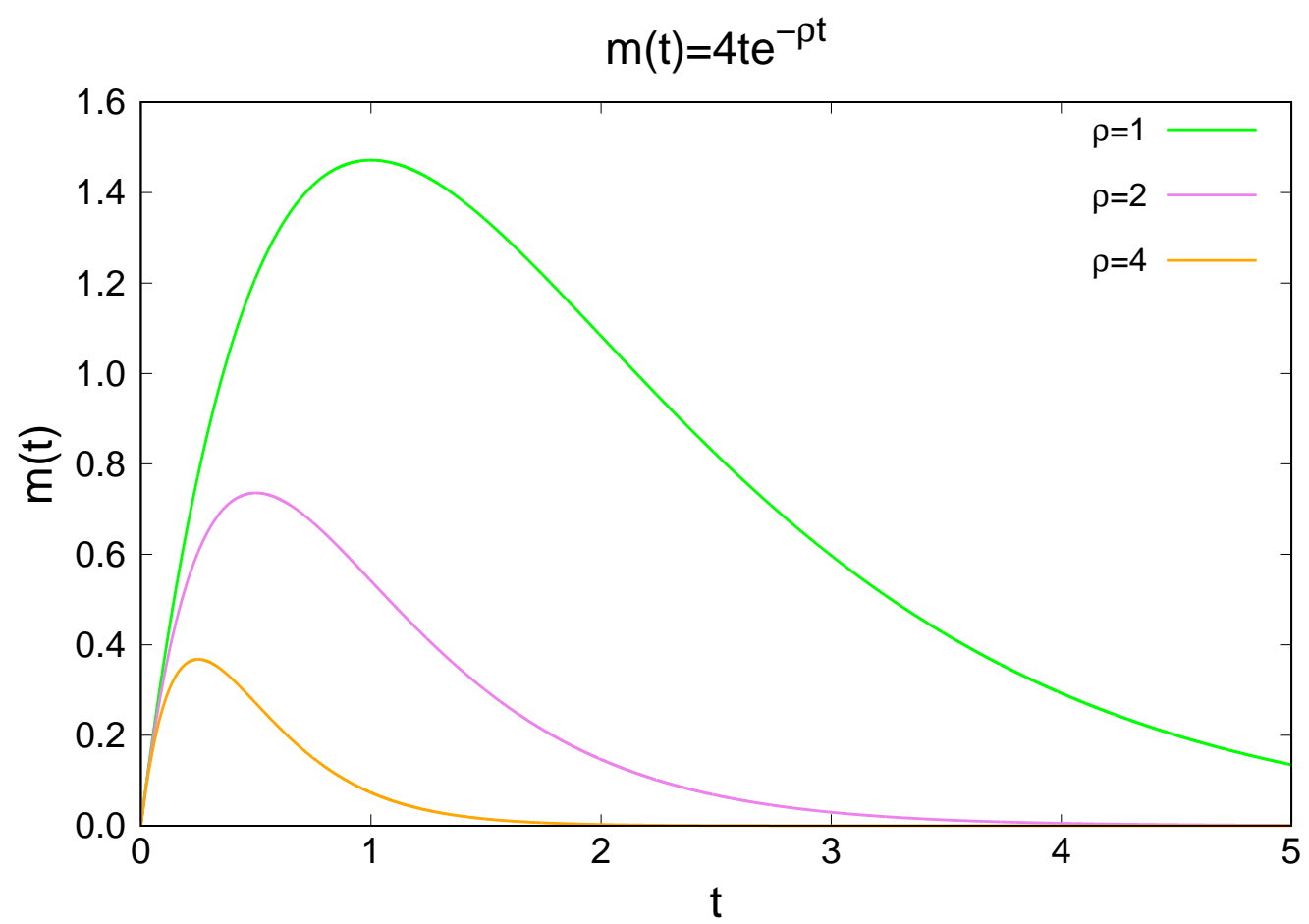

Figure 23. Equation 18 plotted for various values of $\rho$, keeping the other two parameters fixed at $m_{0}=0$ and $c_{0}=4$.

It can be seen graphically from figure 23 that larger $\rho$ results in a quicker peak time for $m(t)$. Subsequently, this means a faster peak time for the $\mathrm{K}^{+}$current during a voltage clamp. In fact, the peak time $t_{\max }$ for $m(t)$ can be found analytically using single-variable calculus:

$$
\begin{aligned}
m^{\prime}\left(t_{\max }\right) & =\left.\frac{d}{d t}\left[e^{-\rho t}\left(c_{0} t+m_{0}\right)\right]\right|_{t=t_{\max }}=0 \\
& =-\rho e^{-\rho t_{\max }}\left(c_{0} t_{\max }+m_{0}\right)+c_{0} e^{-\rho t_{\max }}=0 \\
& =e^{-\rho t_{\max }}\left[-\rho\left(c_{0} t_{\max }+m_{0}\right)+c_{0}\right]=0 \\
& =-\rho c_{0} t_{\max }-\rho m_{0}+c_{0}=0
\end{aligned}
$$


Solving algebraically for $t_{\max }$ yields

$$
\begin{aligned}
t_{\max } & =\frac{c_{0}-\rho m_{0}}{\rho c_{0}} \\
& =\frac{1}{\rho}-\frac{m_{0}}{c_{0}}
\end{aligned}
$$

Equation 20 above gives the most general expression for the peak time $t_{\max }$ of $m(t)$, such that $m(t) \leq m\left(t_{\max }\right)$ for all $t>0$. This expression simplifies when we let $m_{0}=0$, which results in the special case $t_{\max }^{*}=\frac{1}{\rho}$. The case of $m_{0}=0$ results in non-monotone curves for $m(t)$, as can be seen in figure 25 ..

Inserting the general expression for $t_{\max }$ given by equation 20 into the solution for $m(t)$ given by equation 18 results in the value of the maximum $\mathrm{K}^{+}$activation $m_{\max }$ :

$$
\begin{aligned}
m_{\max } & :=m\left(t_{\max }\right) \\
& =e^{-\rho t_{\max }}\left(c_{0} t_{\max }+m_{0}\right) \\
& =e^{-\rho\left(\frac{c_{0}-\rho m_{0}}{\rho c_{0}}\right)}\left[c_{0}\left(\frac{c_{0}-\rho m_{0}}{\rho c_{0}}\right)+m_{0}\right] \\
& =e^{\frac{\rho m_{0}-c_{0}}{c_{0}}}\left(\frac{c_{0}-\rho m_{0}}{\rho}+m_{0}\right)
\end{aligned}
$$

This expression for $m_{\max }$ simplifies greatly when we let $m_{0}=0$, yielding the special case $m_{\max }^{*}=\frac{1}{e} \frac{c_{0}}{\rho}$

Letting $m_{0}=0$ and $\rho=1$ in equation 18 results in the function $m(t)=c_{0} t e^{-t}$, which is plotted for various values of $c_{0}$ in figure 24. On the other hand, letting $c_{0}=1$ and $\rho=1$ yields $m(t)=e^{-t}\left(t+m_{0}\right)$ which is plotted for various $m_{0}$ in figure 25.

Figure 25 shows that $m_{0}$ is a parameter that modulates the monotonicity of the $m(t)$ curve. For large positive values of $m_{0}, m(t)$ is strictly decreasing. For large negative values of $m_{0}$, the curve is strictly increasing. The $m(t)$ curve is non-monotonic when $-2<m_{0}<1$, meaning that it both increases and decreases when $m_{0}$ is held in this range. The green trace for $m(t)$ in figure 25. (for $m_{0}=0$ ) is of great importance here, since scaling it up by the positive factor $g_{K}\left(V-V_{K}\right)$ for $V>V_{K}$ during a voltage clamp results in a $\mathrm{K}^{+}$current $I_{K}$ whose general shape matches that of the active peaked responses sitting on top of a constant leakage current $I_{L}$ in the experimental data of figure 26 . 


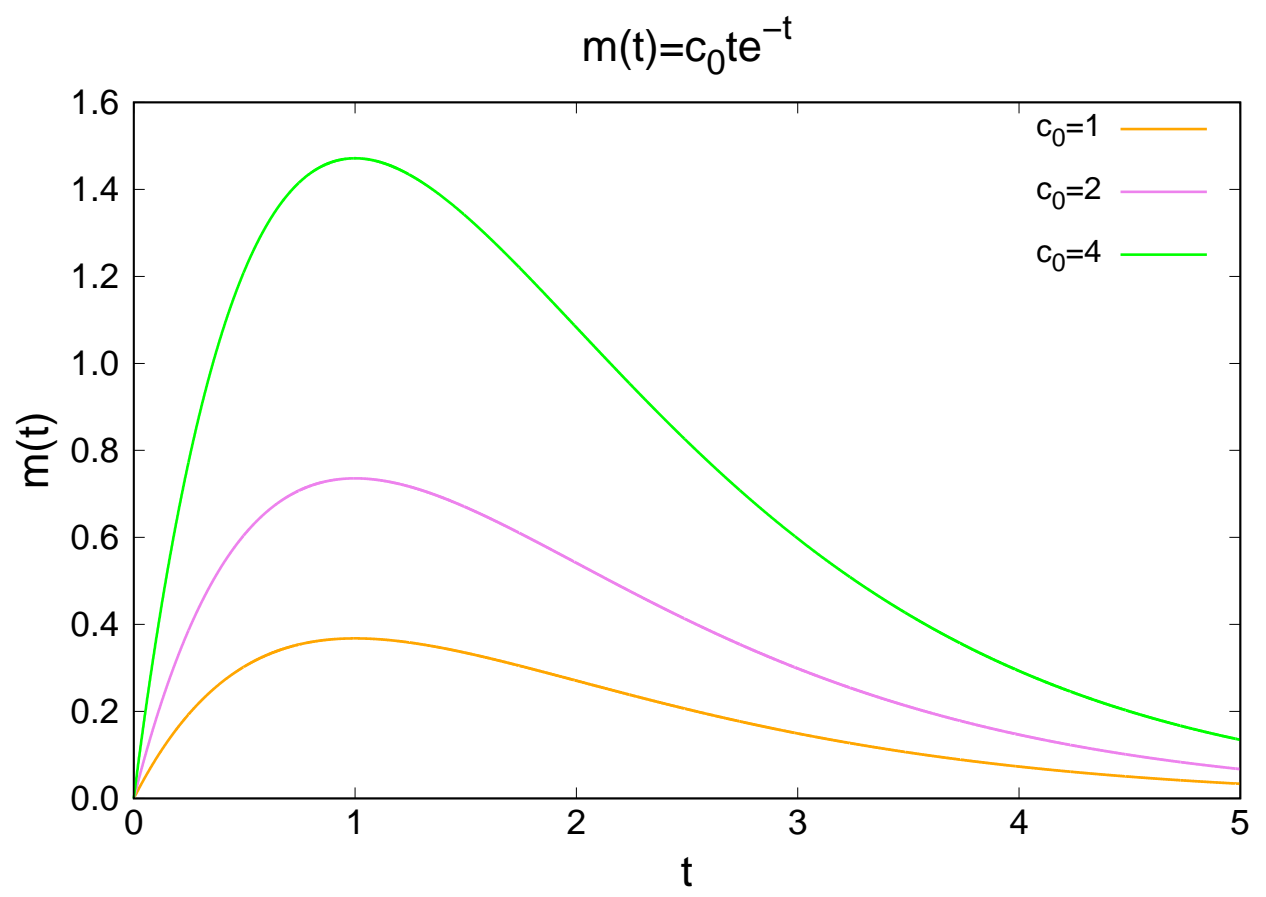

Figure 24. Equation 18 plotted for various values of $c_{0}$, keeping the other two parameters fixed at $m_{0}=0$ and $\rho=1$. Larger $c_{0}$ results in a more peaked response of $m(t)$.

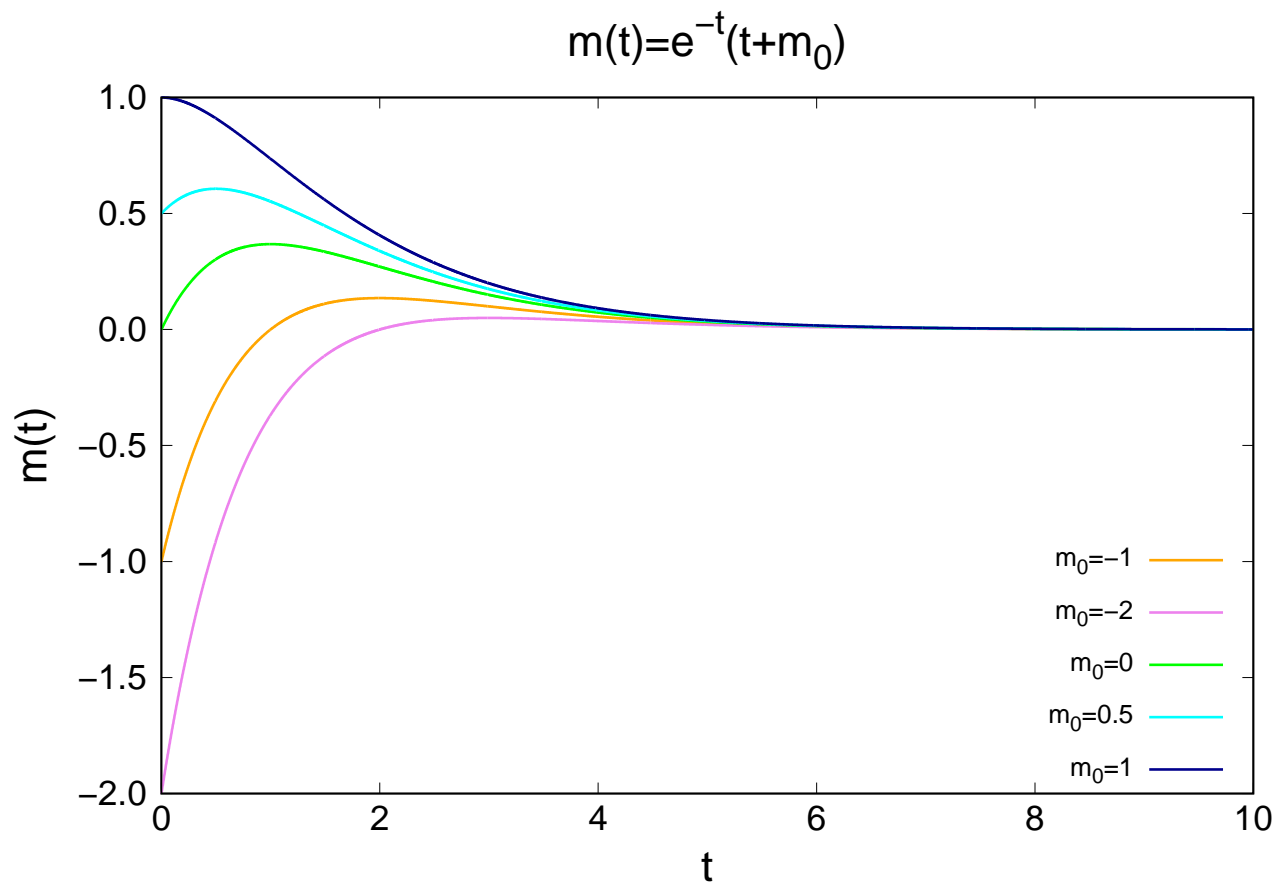

Figure 25. Equation 18 plotted for various values of $m_{0}$, keeping the other two parameters fixed at $c_{0}=1$ and $\rho=1$. 
Using the Steady-State Current to Match the Data

Now that an explicit form has been obtained for both $m(t)$ and $c(t)$, the applied current during a voltage clamp may be written as a function of time. Inserting the solutions given by equations 18 and 19 into the expression $I_{\text {app }}$ given by equation 13 yields

$$
I_{\text {app }}(t)=g_{K}\left[e^{-\rho t}\left(c_{0} t+u_{0}\right)\right]\left(V-V_{K}\right)+g_{L}\left(V-V_{L}\right)
$$

The steady-state of the applied current during a voltage clamp is thus given by

$$
\begin{aligned}
I_{\infty}:=\lim _{t \rightarrow \infty} I_{\mathrm{app}}(t) & =\lim _{t \rightarrow \infty} g_{K}\left[e^{-\rho t}\left(c_{0} t+u_{0}\right)\right]\left(V-V_{K}\right)+g_{L}\left(V-V_{L}\right) \\
I_{\infty} & =g_{L}\left(V-V_{L}\right)
\end{aligned}
$$

Letting $V_{L}=-90 \mathrm{mV}[29]$ and $C=2 \mathrm{pF}$ (as measured in [33]), $g_{L}$ will now be deduced from the experimental data in figure 26. In these data, the AFD neuron is submitted to the same stimulation protocol at two different temperatures. The top plot is at a temperature $T=23.6^{\circ} \mathrm{C}$ and the bottom plot is at a temperature $T=17.7^{\circ} \mathrm{C}$. The cultivation temperature in this experiment is $T_{C}=20^{\circ} \mathrm{C}$. The responses of AFD are measured as the membrane current per unit membrane capacitance in $\mathrm{pA} / \mathrm{pF}$. The stimulation protocol is as follows:

1. The neuron is held at a constant voltage $V=-60 \mathrm{mV}$ during the parts of these traces where the current per unit capacitance $\left(I_{\mathrm{app}} / C_{m}\right.$ in the model $)$ is zero.

2. The neuron is stepped up or down to some other constant voltage during the parts of these traces when $I_{\mathrm{app}} / C_{m} \neq 0$, in $20 \mathrm{mV}$ increments between $-110 \mathrm{mV}$ and $110 \mathrm{mV}$.

There are 7 experimental traces in the top of figure 26. where it is clear that $I_{\mathrm{app}} / C_{m}>0$. From item 2 above, the top trace corresponds to $V=110 \mathrm{mV}$, the next-from-the-top to $V=90 \mathrm{mV}$, and so on, all the way down to the smallest positive trace with the least amount of noise corresponding to $V=-10 \mathrm{mV}$. The trace directly above this corresponds to a voltage clamp of $V=10 \mathrm{mV}$ and has a steady state $I_{\infty} / C_{m} \approx 50 \mathrm{pA} / \mathrm{pF}$. Multiplying this by the membrane capacitance $C_{m}=2 \mathrm{pF}$ yields a steady-state current $I_{\infty}=100 \mathrm{pA}$. Inserting this into equation 24 above with $V=10 \mathrm{mV}$ 
and $V_{L}=-90 \mathrm{mV}$ yields

$$
\begin{aligned}
100 \mathrm{pA} & =g_{L}(10 \mathrm{mV}+90 \mathrm{mV}) \\
g_{L} & =1 \mathrm{nS}
\end{aligned}
$$
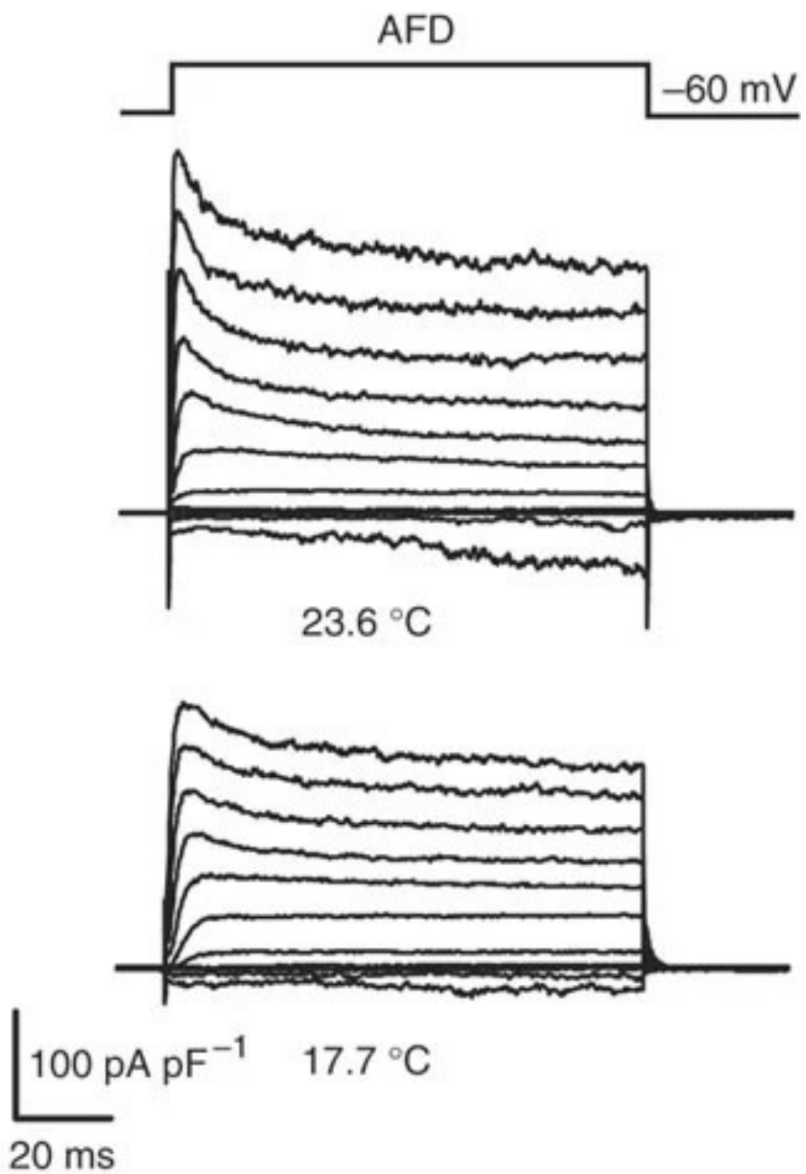

Figure 26. Experimental data from figure 3A of Ramot et al. (2008) [32]. The responses of thermosensory AFD neuron in the form of membrane current per unit capacitance to voltage steps at $17.7^{\circ} \mathrm{C}$ (bottom) and $23.6^{\circ} \mathrm{C}$ (top). See enumerated list on page 29 for details on the stimulation protocol used in this experiment. Reprinted with permission from Springer: Nature Neuroscience [33] Copyright 2008.

Now letting $g_{K}=3 \mathrm{nS}$ and $V_{K}=-80 \mathrm{mV}$, a model result is shown in figure 27 . below that compares well to the experimental traces in the top of figure 26. A model result is shown in figure 28 below that compares well to the experimental traces in the bottom of figure 26 . These results indicate that $c_{0}$ is an increasing function of voltage whose slope is decreased at cooler temperatures. Figures 27 and 28 also show that there exists some threshold voltage, above which, $m_{0}=0$. Below 
this threshold, $m_{0}$ is negative and an increasing function of voltage. This function $m_{0}=m_{0}(V)$ with associated threshold is downshifted at higher temperatures.

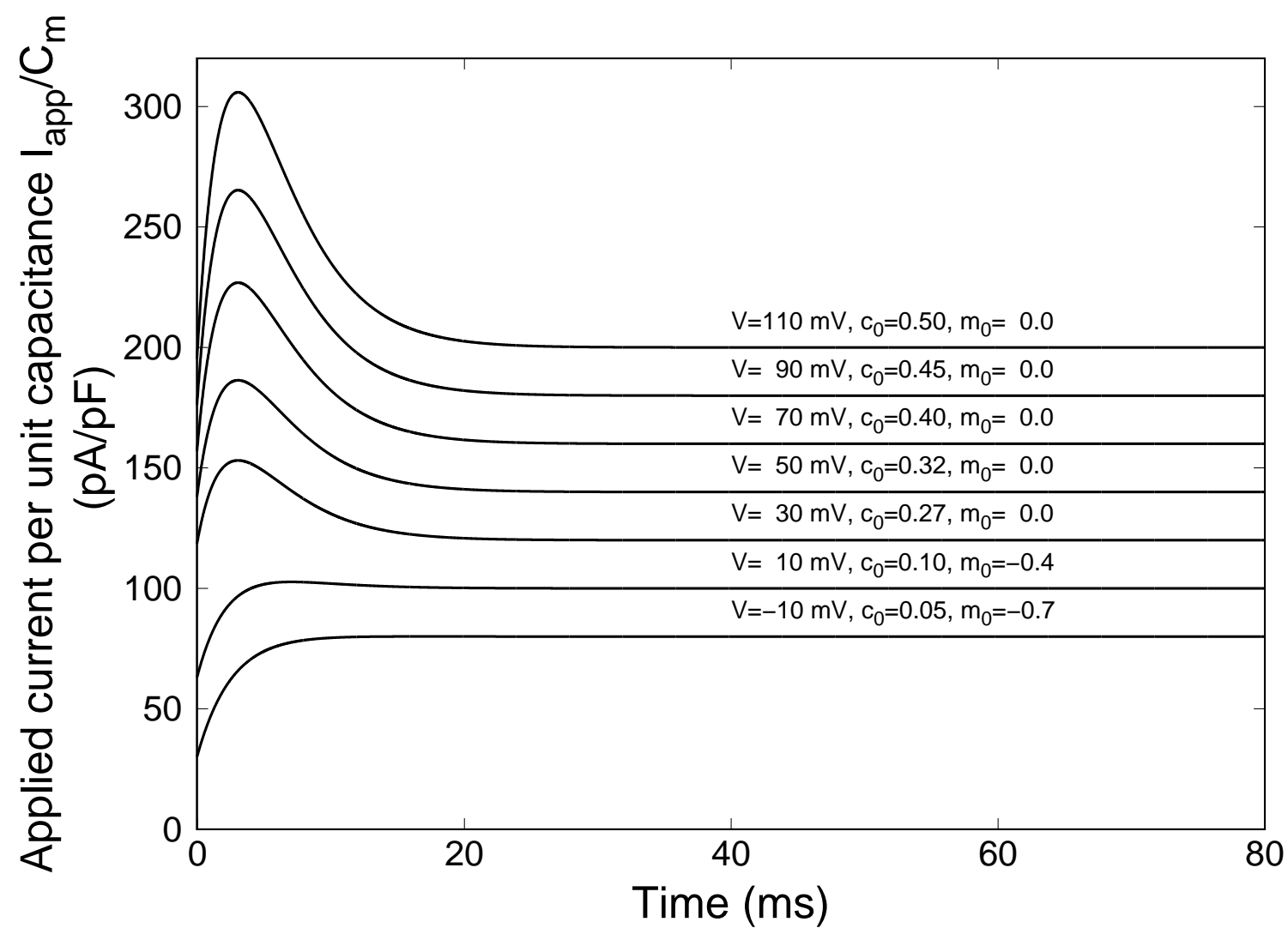

Figure 27. Theoretical current responses (given by equation 22 divided by $C_{m}=2 \mathrm{pF}$ ) during various voltage clamps, to serve as a comparison with the time series traces in the top of figure 26 . $\rho=0.33 \mathrm{~ms}^{-1}$ for all traces here, yielding a peak time of $t_{\max }^{*} \approx 3 \mathrm{~ms}$ for all traces with $V>10$ $\mathrm{mV}$. Nernst potentials are set at $V_{K}=-80 \mathrm{mV}$ and $V_{L}=-90 \mathrm{mV}$.

The curves in figure 27 above match qualitatively, and somewhat quantitatively, the experimental curves in the top of figure 26 taken at $T=23.6^{\circ} \mathrm{C}$. Similarly, the curves in figure 27 match the bottom of figure 26 , taken at $T=17.7^{\circ} \mathrm{C}$. In each case, this was achieved by manually adjusting the parameters $c_{0}, m_{0}$, and $\rho$ to acquire curves similar to that shown in the experiment. The voltage parameter $V$ was defined explicitly from the experiment. The model predicts that $\rho$ is constant when $T$ is constant, reinforcing the notion that $\rho$ is solely a function of temperature. 


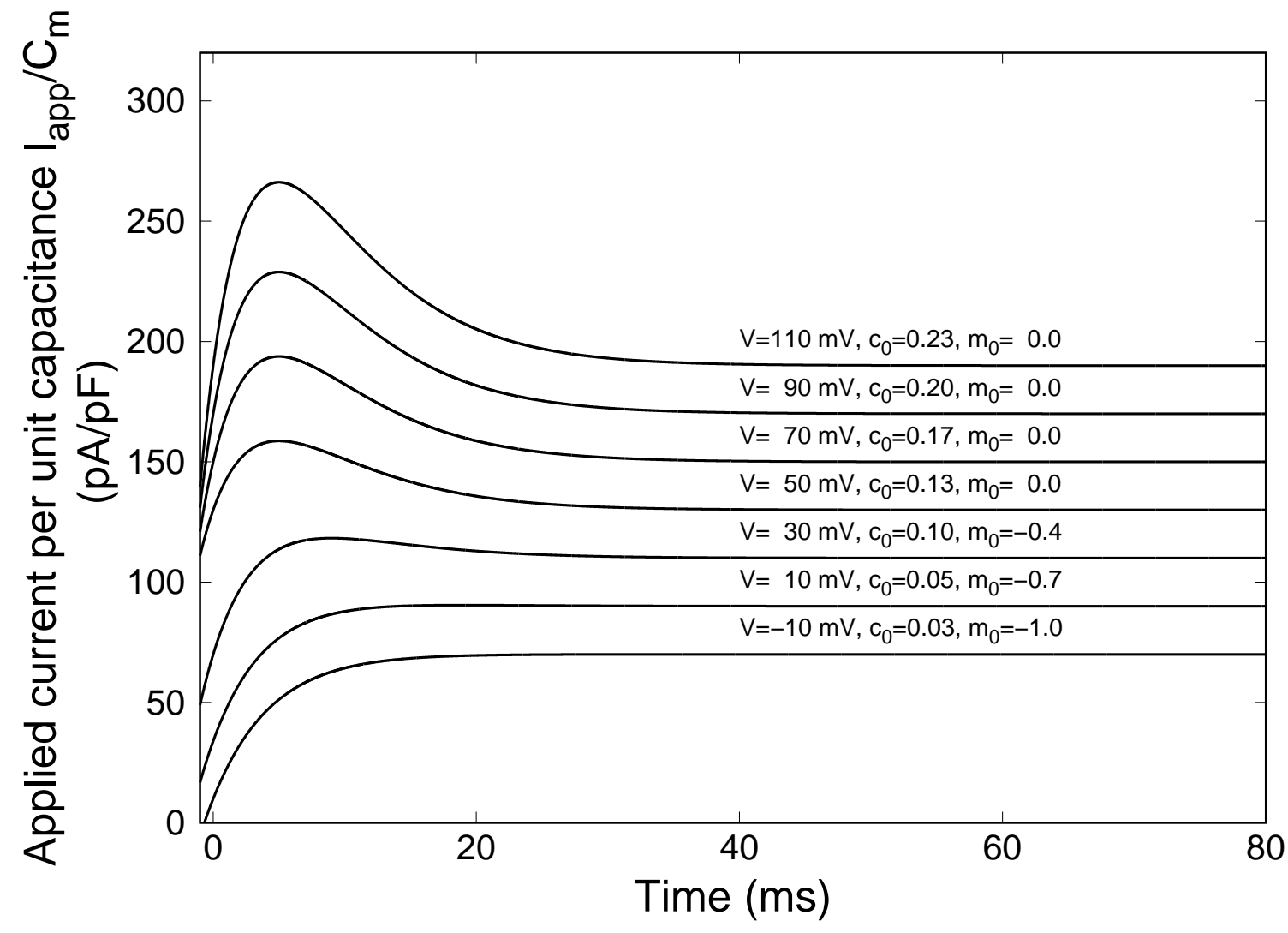

Figure 28. Theoretical current responses (given by equation 22 divided by $C_{m}=2 \mathrm{pF}$ ) during various voltage clamps, to serve as a comparison with the time series traces in the bottom of figure 26.. $\rho=0.2 \mathrm{~ms}^{-1}$ for all traces here, yielding a peak time of $t_{\max }^{*}=5 \mathrm{~ms}$ for all traces with $V>30$ $\mathrm{mV}$. Nernst potentials are set at $V_{K}=-70 \mathrm{mV}$ and $V_{L}=-80 \mathrm{mV}$.

The Nernst potential of an ion $S$ is given by the equation

$$
V_{S}=\frac{k T}{z q} \ln \left(\frac{[S]_{e}}{[S]_{i}}\right)
$$

where $k$ is Boltzmann's constant, $T$ is the absolute temperature, $z$ is the ionic charge, $q$ is the charge of a proton, $[S]_{e}$ is the extracellular concentration of ion $S$, and $[S]_{i}$ is the intracellular concentration [23]. Assuming that the ratio of concentrations $[S]_{e} /[S]_{i}<1$ changes negligibly for the potassium and leak ions, temperature is the only parameter affecting the Nernst potentials. At lower temperatures, the magnitude of $V_{S}$ is lower. This is the justification for why the magnitude of their respective Nernst potentials are set $10^{\circ} \mathrm{C}$ lower in figure 28 than they are in figure 27 .. 


\section{CHAPTER IV: CONCLUSIONS}

\section{Calcium}

The parameters of the original calcium model, as devised by Kuramochi \& Doi in 2017, were fit using data from the chemo-sensitive ASER neuron. Given that these parameters were changed minimally in the AFD model results here, it is reasonable to claim that the mechanism by which ASER $\left[\mathrm{Ca}^{2+}\right]$ activates in response to fluctuations in $\mathrm{NaCl}$ has similarities to that of the mechanism by which AFD activates to temperature changes. Indeed, experimental research supports this claim since both ASER and AFD are known to rely on a common family of transmembrane proteins, the receptor guanylate cyclases (rGC's), to mediate their responses to $\mathrm{NaCl}$ and temperature, respectively [30],[21]. The rGC's in AFD and ASER implicated in thermosensation and chemosensation, respectively, are genetically distinct. However, they are both known to activate a second messenger signaling cascade mediated by cyclic guanosine monophosphate (cGMP). A sufficient change in intracellular cGMP concentration subsequently triggers a change in permeability of the cGMP-gated nonselective cation channel encoded by the tax-2 and tax-4 genes [24]. The results of the phenomenological modeling done here suggest that the sensory transduction mechanism by which temperature oscillations cause phase-locked oscillations in AFD $\left[\mathrm{Ca}^{2+}\right]$ may be analogous to the mechanism by which periodic fluctuations in extracellular $\mathrm{NaCl}$ cause phase-locked oscillations in ASER $\left[\mathrm{Ca}^{2+}\right]$. Given the commonalities between the molecules underlying these two different processes, such a speculation is not unreasonable.

In figures 16,18 , and 20 , the activation of $I(t)$ is conditional on temperature being above a certain value, $T_{C}-4$. Deregulation of the threshold temperature associated with thermoreceptor currents is seen upon genetic deletion of the phosphodiesterase-2 (PDE-2) in AFD [42], which the phenomological model does not capture directly. Fortunately, $I(t)$ is a dimensionless quantity and can be interpreted loosely as any stimulus that causes an increase in dendritic calcium levels. While the present implementation of the parameters $\rho$ and $\phi$ cannot replace $I(t)$ as a stimulus that

creates transient $\mathrm{Ca}^{2+}$ responses, they do provide a simple framework for tuning the temperature sensitivity of the model neuron.

Of particular significance, the peak time results of the calcium model form a nice parallel 
with the assymmetry in the behavior of $C$. elegans, with respect to temperature. As mentioned previously, the worm can only respond to temperature changes within a critical time window of 20 seconds [41]. If it is assumed that the peak time of a calcium response by AFD is roughly coincident with the timing of the subsequent behavior it demands, then figure 13 shows that model neurons with high $\rho_{0}$ ( 4 or 5 , for example) values have dendrite peak times that are greater than 20 seconds for low temperatures (less than $14^{\circ} \mathrm{C}$, for example). This is consistent with the behavioral studies that have shown the worm to be much less successful at thermotaxis when ambient temperature is much lower than the cultivation temperature $\left(T<<T_{C}\right)$, compared to when it is much higher $\left(T>>T_{C}\right)[18][20]$.

\section{Membrane Electrodynamics}

Of immediate concern is the interpretation of the variable " $c$ " in the membrane current model. If one of the primary mediators of ionic currents in the AFD neuronal membrane during a voltage clamp is a group of calcium-regulated potassium channels, then $c$ may be appropriately interpreted as the calcium concentration in the immediate vicinity of these potassium channels. The prediction of exponential decay from some initial value implicitly assumes that there is no active calcium production surrounding these potassium channels at the nano-meter spatial scale and milli-second time scale, during a voltage clamp. Given that there are no experimental studies (to the best of the author's knowledge, at the time of writing) simultaneously monitoring $\left[\mathrm{Ca}^{2+}\right]$ and membrane voltage/current of AFD during a temperature stimulus, this prediction cannot be rejected nor confirmed.

An alternative candidate for the chemical origin of $c$ in the membrane electrodynamics model is that of cGMP (cyclic guanosine monophosphate) which is a cyclic nucleotide that gates the cationselective TAX- $4 / 2$ channels of the worm's neuronal membranes. The TAX-4 channels are found in both chemosensory and thermosensory neurons in C. elegans, and are subsqeuently necessary for both chemotaxis and thermotaxis [24]. Unique to the AFD thermosensory neurons are a set of genes that encode for three distinct guanylate cyclase proteins termed GCY-8, GCY-18, and GCY-23. It is hypothesized that these membrane proteins respond to temperature stimuli by increasing the intracellular cGMP concentration, which in turn increases the conductance of the TAX-4 channel, 
hence allowing cations to flow and subsequently altering the membrane voltage of AFD [21]. If we suppose that warmer temperatures result in larger cGMP concentrations, then the relationship between $c_{0}$ and $V$ at the two different temperatures of figures 27 and 28 may be conceptualized as in figure 29 below. It is the increase in slope of the $c_{0}-V$ relationship at higher temperatures which results in the more "peaked" reponses of figure 27 , relative to figure 28 . This is consistent with results from a recent fluorescence study showing that cGMP levels in AFD during a positive temperature ramp are higher when the ramp is higher [44]. Amplification of the membrane current at higher temperatures like this is potentially a dynamical distinction of AFD from other C. elegans neurons.

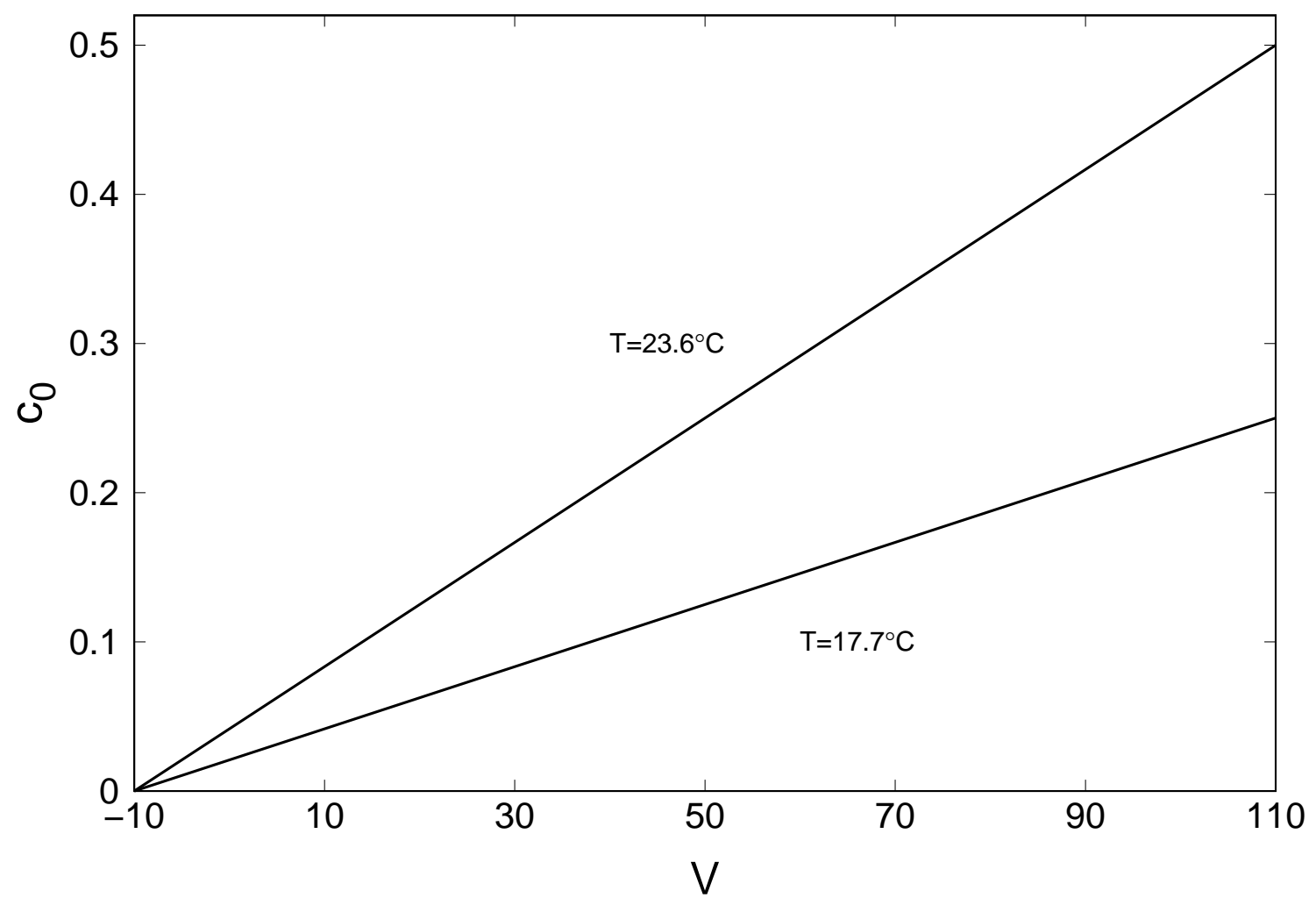

Figure 29. Relationship between initial intracellular cGMP concentration $c_{0}$ and constant membrane voltage $V$ at two different constant temperatures. 
Broad Conclusions, Caveats, and Future Outlook

A conscious decision by the modeler was made in figure 16. to synchronize the oscillations of input $I(t)$ with the temperature oscillations, in order to acquire the synchronous calcium response. The AFD neuron may be making a similar "decision" when phase-locking its response with small temperature oscillations. Such temperature oscillations would inflict naturally upon AFD if the hosting worm were crawling in a sufficiently steep thermal gradient, because of the back-and-forth motion it makes with its head. How the resulting $\left[\mathrm{Ca}^{2+}\right]$ oscillations arise mechanistically is an open question. The results of the calcium model simulated in chapter II here suggest that whatever mechanism ASER uses for chemoreception of repetitive salt pulses with synchronous $\left[\mathrm{Ca}^{2+}\right]$ oscillations has similarities to AFD's mechanism for doing the same with temperature oscillations. An alternative is that suggested by the supplementary model simulated in figure 31 of Appendix B. This result shows that a constant input $(\beta)$ can cause oscillations in $\left[\mathrm{Ca}^{2+}\right]$ with the same approximate period as that shown experimentally in figure 17.

In the oscillatory case, the AFD thermotransduction machinery computes the first derivative of the temperature signal above some threshold temperature, and outputs this (with some phase lag) as the $\left[\mathrm{Ca}^{2+}\right]$. In fact, this theory is consistent with the non-oscillatory case when $T$ stays below $T_{C}$, as well. When $T$ goes above $T_{C}$, the results of Ramot et al. (2008), shown here on the right of figure 33, become illuminating when interpreted in conjunction with the calcium data. They show that there is a large and fast spike in membrane current when temperature passes the cultivation temperature of $T_{C}=20^{\circ} \mathrm{C}$. This current is likely carried by inward flowing $\mathrm{Ca}^{2+}$, since the fluorescence data by Yoshida et al. (2016) displayed here in figure 21 demonstrate that there is a large increase in AFD's $\left[\mathrm{Ca}^{2+}\right]$ during this same event. This suggests that a correct model of the AFD's membrane currents during a temperature stimulus would include a calcium current that activates when ambient temperature surpasses some threshold. An alternative hypothesis is that this depolarizing current causes a large release of calcium from internal stores within the AFD neuron. Perhaps both are true, to some extent.

The membrane electrodynamics model was constructed with the intent to match data taken at constant temperature and constant membrane voltage. It is true, however, that both of these variables are changing during thermotaxis (see figure 33 in Appendix $\mathrm{C}$ ). How the membrane 
electrodynamics model can be extended to describe situations where the temperature is changing is a question worth investigating. It may require adding currents that are sensitive to the derivative of temperature, or ones that simply encode whether or not the threshold temperature has been reached. The supplementary modeling done in Appendix $\mathrm{C}$ is a first attempt at tackling this mystery.

The AFD neuron was studied in isolation here, to gain a better understanding of its individual role in thermoreception. It should be realized, however, that this cell is but one node in a larger network. This is illustrated in figure 30 where the synaptic partners of AFD are sketched with their weighted connections. Recalling that the neuron model of Kuramochi \& Doi (2017) sought to describe ASE, it is no surprise that an adapted version of this model performed reasonably well at describing AFD, given the fact that these two neurons share a bidirectional synapse. Of particular relevance to the behavior of thermotaxis, the interneuron AIY shares a synapse with a downstream neuron AIZ, both of which are implicated in thermotaxis. Killing AIY with a laser results in cryophilic (abnormally cold-seeking) worms that cannot track isotherms; killing AIZ with a laser results in thermophilic (abnormally heat-seeking) worms [26]. AFD communicates unidirectionally with AIY through a chemical synapse, whose activity is likely coupled to the intracellular calcium dynamics of AFD. Future modeling studies aimed at incorporating the results shown here with the network picture of figure 30 will need to decide on a set of laws governing the synapses between AFD, AIY, and AIZ that is consistent with their roles observed in laser ablation or genetic manipulation studies. It is not clear how $\left[\mathrm{Ca}^{2+}\right]$ oscillations in AFD propagate through this thermosensory circuit, eventually to inform downstream motor neurons of the correct movement pattern.

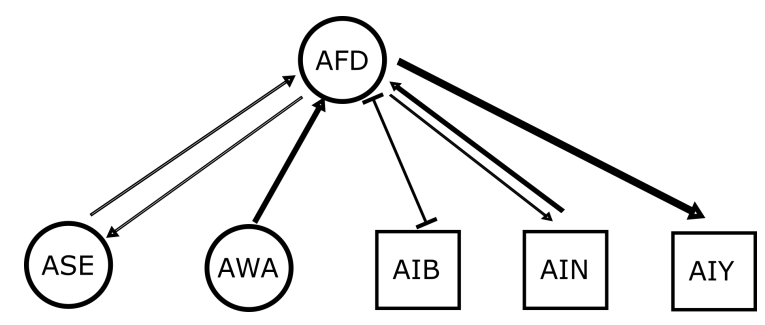

Figure 30. Local network of AFD with its synaptic partners. Circles represent sensory neurons. Squares represent interneurons. Arrows represent chemical synapses. Lines with cross bars on either end represent electrical synapses. Thicker lines mean stronger synapses. Based on data from [43]. 


\section{REFERENCES}

[1] Z.F Altun and D.H. Hall. Nervous system, general description. WormAtlas, 2003.

[2] Sandra Batten. Climate change and the macro-economy: a critical review. 2018.

[3] Francis Gano Benedict, Walter Richard Miles, and Alice Johnson. The temperature of the human skin. Proceedings of the national academy of sciences of the United States of America, $5(6): 218,1919$.

[4] Sydney Brenner. The genetics of Caenorhabditis elegans. Genetics, 77(1):71-94, 1974.

[5] Martin Chalfie, Yuan Tu, Ghia Euskirchen, William W Ward, and Douglas C Prasher. Green fluorescent protein as a marker for gene expression. Science, 263(5148):802-805, 1994.

[6] Damon A Clark, David Biron, Piali Sengupta, and Aravinthan DT Samuel. The AFD sensory neurons encode multiple functions underlying thermotactic behavior in Caenorhabditis elegans. Journal of Neuroscience, 26(28):7444-7451, 2006.

[7] Ann K Corsi, Bruce Wightman, and Martin Chalfie. A transparent window into biology: a primer on Caenorhabditis elegans. Genetics, 200(2):387-407, 2015.

[8] Fábio M DaMatta and José D Cochicho Ramalho. Impacts of drought and temperature stress on coffee physiology and production: a review. Brazilian journal of plant physiology, 18(1):5581, 2006.

[9] Shaina Dhamija and Arijit K De. Elucidating Contributions from Multiple Species during Photoconversion of Enhanced Green Fluorescent Protein (EGFP) under Ultraviolet Illumination. Photochemistry and Photobiology, 2021.

[10] Ellsworth C Dougherty and Hermione Grant Calhoun. Possible significance of free-living nematodes in genetic research. Nature, 161(4079):29-29, 1948.

[11] Marie-Anne Félix, Alyson Ashe, Joséphine Piffaretti, Guang Wu, Isabelle Nuez, Tony Bélicard, Yanfang Jiang, Guoyan Zhao, Carl J Franz, Leonard D Goldstein, et al. Natural and experimental infection of Caenorhabditis nematodes by novel viruses related to nodaviruses. PLoS Biol, 9(1):e1000586, 2011. 
[12] Howard Ferris and WF Hieb. Ellsworth C. Dougherty: a pioneer in the selection of Caenorhabditis elegans as a model organism. Genetics, 200(4):991-1002, 2015.

[13] Christian Finke, Jan A Freund, Epaminondas Rosa Jr, Paul H Bryant, Hans A Braun, and Ulrike Feudel. Temperature-dependent stochastic dynamics of the Huber-Braun neuron model. Chaos: An Interdisciplinary Journal of Nonlinear Science, 21(4):047510, 2011.

[14] Elahe Ganji, C Savio Chan, Christopher W Ward, and Megan L Killian. Optogenetic activation of muscle contraction in vivo. Connective tissue research, 62(1):15-23, 2021.

[15] Albert Goldbeter, Geneviéve Dupont, and Michael J Berridge. Minimal model for signalinduced ca2+ oscillations and for their frequency encoding through protein phosphorylation. Proceedings of the National Academy of Sciences, 87(4):1461-1465, 1990.

[16] James D Hardy. Physiology of temperature regulation. Physiological Reviews, 41(3):521-606, 1961.

[17] Edward M Hedgecock, Joseph G Culotti, and David H Hall. The unc-5, unc-6, and unc-40 genes guide circumferential migrations of pioneer axons and mesodermal cells on the epidermis in C. elegans. Neuron, 4(1):61-85, 1990.

[18] Edward M Hedgecock and Richard L Russell. Normal and mutant thermotaxis in the nematode Caenorhabditis elegans. Proceedings of the National Academy of Sciences, 72(10):4061-4065, 1975.

[19] Raymond B Huey. Temperature, physiology, and the ecology of reptiles. In Biology of the Reptilia. Citeseer, 1982.

[20] Muneki Ikeda, Shunji Nakano, Andrew C Giles, Linghuan Xu, Wagner Steuer Costa, Alexander Gottschalk, and Ikue Mori. Context-dependent operation of neural circuits underlies a navigation behavior in Caenorhabditis elegans. Proceedings of the National Academy of Sciences, 117(11):6178-6188, 2020.

[21] Hitoshi Inada, Hiroko Ito, John Satterlee, Piali Sengupta, Kunihiro Matsumoto, and Ikue Mori. Identification of guanylyl cyclases that function in thermosensory neurons of Caenorhabditis elegans. Genetics, 172(4):2239-2252, 2006. 
[22] Eugene M Izhikevich. Dynamical systems in neuroscience. MIT press, 2007.

[23] James P Keener and James Sneyd. Mathematical physiology, volume 1. Springer, 1998.

[24] Hidetoshi Komatsu, Ikue Mori, Jeong-Seop Rhee, Norio Akaike, and Yasumi Ohshima. Mutations in a cyclic nucleotide-gated channel lead to abnormal thermosensation and chemosensation in C. elegans. Neuron, 17(4):707-718, 1996.

[25] Masahiro Kuramochi and Motomichi Doi. A computational model based on multi-regional calcium imaging represents the spatio-temporal dynamics in a Caenorhabditis elegans sensory neuron. PLoS One, 12(1):e0168415, 2017.

[26] Ikue Mori and Yasumi Ohshima. Neural regulation of thermotaxis in Caenorhabditis elegans. Nature, 376(6538):344-348, 1995.

[27] Georg Nagel, Martin Brauner, Jana F Liewald, Nona Adeishvili, Ernst Bamberg, and Alexander Gottschalk. Light activation of channelrhodopsin-2 in excitable cells of Caenorhabditis elegans triggers rapid behavioral responses. Current Biology, 15(24):2279-2284, 2005.

[28] Phuong Anh T Nguyen, Willisa Liou, David H Hall, and Michel R Leroux. Ciliopathy proteins establish a bipartite signaling compartment in a c. elegans thermosensory neuron. Journal of cell science, 127(24):5317-5330, 2014.

[29] Martina Nicoletti, Alessandro Loppini, Letizia Chiodo, Viola Folli, Giancarlo Ruocco, and Simonetta Filippi. Biophysical modeling of C. elegans neurons: Single ion currents and wholecell dynamics of $\mathrm{AWC}^{\text {on }}$ and RMD. PloS one, 14(7):e0218738, 2019.

[30] Christopher O Ortiz, Serge Faumont, Jun Takayama, Heidi K Ahmed, Andrew D Goldsmith, Roger Pocock, Kathryn E McCormick, Hirofumi Kunimoto, Yuichi Iino, Shawn Lockery, et al. Lateralized gustatory behavior of c. elegans is controlled by specific receptor-type guanylyl cyclases. Current Biology, 19(12):996-1004, 2009.

[31] PV Vara Prasad, SR Pisipati, Z Ristic, U Bukovnik, and AK Fritz. Impact of nighttime temperature on physiology and growth of spring wheat. Crop science, 48(6):2372-2380, 2008. 
[32] Daniel Ramot, Bronwyn L MacInnis, and Miriam B Goodman. Bidirectional temperaturesensing by a single thermosensory neuron in C. elegans. Nature neuroscience, 11(8):908, 2008.

[33] Renaud Renault, Nirit Sukenik, Stéphanie Descroix, Laurent Malaquin, Jean-Louis Viovy, Jean-Michel Peyrin, Samuel Bottani, Pascal Monceau, Elisha Moses, and Maéva Vignes. Combining microfluidics, optogenetics and calcium imaging to study neuronal communication in vitro. PloS one, 10(4):e0120680, 2015.

[34] George H Rutherford, Zach D Mobille, Jordan Brandt-Trainer, Rosangela Follmann, and Epaminondas Rosa Jr. Analog implementation of a hodgkin-huxley model neuron. American Journal of Physics, 88(11):918-923, 2020.

[35] Tapio Schneider and Isaac M Held. Discriminants of twentieth-century changes in earth surface temperatures. Journal of Climate, 14(3):249-254, 2001.

[36] Piali Sengupta, Heather A Colbert, and Cornelia I Bargmann. The C. elegans gene odr-7 encodes an olfactory-specific member of the nuclear receptor superfamily. Cell, 79(6):971-980, 1994.

[37] Mark Shaaya, Jordan Fauser, and Andrei V Karginov. Optogenetics: the art of illuminating complex signaling pathways. Physiology, 36(1):52-60, 2021.

[38] Aakanksha Singhvi, Bingqian Liu, Christine J Friedman, Jennifer Fong, Yun Lu, Xin-Yun Huang, and Shai Shaham. A glial k/cl transporter controls neuronal receptive ending shape by chloride inhibition of an rgc. Cell, 165(4):936-948, 2016.

[39] John E Sulston, Einhard Schierenberg, John G White, J Nichol Thomson, et al. The embryonic cell lineage of the nematode Caenorhabditis elegans. Developmental Biology, 100(1):64-119, 1983.

[40] C. elegans Sequencing Consortium*. Genome sequence of the nematode C. elegans: a platform for investigating biology. Science, 282(5396):2012-2018, 1998. 
[41] Yuki Tsukada, Masataka Yamao, Honda Naoki, Tomoyasu Shimowada, Noriyuki Ohnishi, Atsushi Kuhara, Shin Ishii, and Ikue Mori. Reconstruction of spatial thermal gradient encoded in thermosensory neuron AFD in Caenorhabditis elegans. Journal of Neuroscience, 36(9):2571$2581,2016$.

[42] Dong Wang, Damien O'Halloran, and Miriam B Goodman. GCY-8, PDE-2, and NCS-1 are critical elements of the cGMP-dependent thermotransduction cascade in the AFD neurons responsible for C. elegans thermotaxis. Journal of General Physiology, 142(4):437-449, 2013.

[43] John G White, Eileen Southgate, J Nichol Thomson, and Sydney Brenner. The structure of the nervous system of the nematode Caenorhabditis elegans. Philos Trans R Soc Lond B Biol Sci, 314(1165):1-340, 1986.

[44] Sarah Woldemariam, Jatin Nagpal, Tyler Hill, Joy Li, Martin W Schneider, Raakhee Shankar, Mary Futey, Aruna Varshney, Nebat Ali, Jordan Mitchell, et al. Using a robust and sensitive gfp-based cgmp sensor for real-time imaging in intact caenorhabditis elegans. Genetics, 213(1):59-77, 2019.

[45] A Yoshida, S Nakano, T Suzuki, K Ihara, T Higashiyama, and I Mori. A glial K+/Cl- cotransporter modifies temperature-evoked dynamics in Caenorhabditis elegans sensory neurons. Genes, Brain and Behavior, 15(4):429-440, 2016.

[46] Gabriel Yvon-Durocher, Andrew P Allen, David Bastviken, Ralf Conrad, Cristian Gudasz, Annick St-Pierre, Nguyen Thanh-Duc, and Paul A Del Giorgio. Methane fluxes show consistent temperature dependence across microbial to ecosystem scales. Nature, 507(7493):488-491, 2014 .

[47] L.A. Herndon Z.F. Altun. Wormatlas. http://www.wormatlas.org, 2002-2021. Accessed: 2021-03-09. 
APPENDIX A: VARIABLES $W_{d}$ AND $W_{a}$ (ADAPTED FROM [25])

\begin{tabular}{|c|c|c|c|}
\hline & $\boldsymbol{x}_{\boldsymbol{a}}>\mathbf{0}$ & $\boldsymbol{x}_{\boldsymbol{a}}<\mathbf{0}$ & $\boldsymbol{x}_{\boldsymbol{a}}=\mathbf{0}$ \\
\hline \multirow{3}{*}{$\boldsymbol{x}_{\boldsymbol{d}}>\mathbf{0}$} & $W_{d}=\frac{\left|x_{a}\right|}{\left|x_{d}+x_{a}\right|}$ & $W_{d}=0$ & $W_{d}=\frac{\left|x_{a}\right|}{\left|x_{d}+x_{a}\right|}$ \\
& $W_{a}=\frac{\left|x_{d}\right|}{\left|x_{d}+x_{a}\right|}$ & $W_{a}=1$ & $W_{a}=\frac{\left|x_{d}\right|}{\left|x_{d}+x_{a}\right|}$ \\
\hline \multirow{2}{*}{$\boldsymbol{x}_{\boldsymbol{d}}<\mathbf{0}$} & $W_{d}=1$ & $W_{d}=\frac{\left|x_{d}\right|}{\left|x_{d}+x_{a}\right|}$ & $W_{d}=1$ \\
& $W_{a}=0$ & $W_{a}=\frac{\left|x_{a}\right|}{\left|x_{d}+x_{a}\right|}$ & $W_{a}=0$ \\
\hline \multirow{2}{*}{$\boldsymbol{x}_{\boldsymbol{d}}=\mathbf{0}$} & $W_{d}=0$ & $W_{d}=0$ & $W_{d}=0$ \\
& $W_{a}=1$ & $W_{a}=1$ & $W_{a}=0$ \\
\hline
\end{tabular}

Table 1. Description of weighting variables $W_{d}$ and $W_{a}$ in the intracellular calcium model stated by equations $1-7$. 


\section{APPENDIX B: GOLDBETER-DUPONT-BERRIDGE $\mathrm{CA}^{2+}$ MODEL}

The minimal model published by Goldbeter and colleagues [15] is used here here to account for the temperature-induced $\mathrm{Ca}^{2+}$ oscillations seen in the AFD neurons. It is stated as follows:

$$
\begin{aligned}
\frac{d c}{d t} & =v_{0}+v_{1} \beta-v_{2}+v_{3}+k_{f} c_{E R}-k c \\
\frac{d c_{E R}}{d t} & =v_{2}-v_{3}-k_{f} c \\
v_{2} & =V_{M 2} \frac{c^{n}}{K_{2}^{n}+c^{n}} \\
v_{3} & =V_{M 3}\left(\frac{c_{E R}^{m}}{K_{R}^{m}+c_{E R}^{m}}\right)\left(\frac{c^{p}}{K_{A}^{p}+c^{p}}\right)
\end{aligned}
$$

where $t$ is time in seconds. $c$ and $c_{E R}$ are the calcium ion concentrations in the cytosol and endoplasmic reticulum (ER), respectively. $v_{0}$ is the constant rate of $\mathrm{Ca}^{2+}$ influx to the cytosol from the outside of the cell. $k c$ is the rate of $\mathrm{Ca}^{2+}$ efflux from the cytosol to the extracellular space. $v_{1}$ is the rate at which $\mathrm{Ca}^{2+}$ is released from an intracellular compartment that is sensitive to Inositol 1,4,5-triphosphate $\left(\mathrm{IP}_{3}\right) . v_{2}$ is the rate with which $\mathrm{Ca}^{2+}$ is pumped from the cytosol into the ER, which is an $\mathrm{IP}_{3}$-insensitive compartment. $v_{3}$ is the rate with which $\mathrm{Ca}^{2+}$ is pumped from the ER back into the cytosol. $k_{f} c_{E R}$ is a constant leakage of $\mathrm{Ca}^{2+}$ from the ER into the cytosol. $V_{M 2}$ and $V_{M 3}$ are the maximum rates of $\mathrm{Ca}^{2+}$ pumping to and fro the ER, respectively. $n$ and $m$ are the corresponding Hill coefficients and $p$ describes how cooperative the activation is. The pumping, release, and activation threshold constants are captured by $K_{2}, K_{R}$, and $K_{A}$, respectively.

The result shown below in figure 2931 is a simulation of equations 26-29, where a constant upstep in the saturation factor $\beta$ causes periodic $\left[\mathrm{Ca}^{2+}\right]$ oscillations in a model cell. While temperature is not accounted for explicitly in this supplementary model, the period of the $\left[\mathrm{Ca}^{2+}\right]$ spikes $(\approx$ 20 seconds) matches that of the $\left[\mathrm{Ca}^{2+}\right]$ spikes in the experimental data shown in figure 31..The parameter values used in figure 31 are given in table 2. 


\begin{tabular}{|c|c|}
\hline Parameter & Value \\
\hline$v_{0}$ & 0.04 \\
\hline$v_{1}$ & 0.292 \\
\hline$V_{M 2}$ & 2.6 \\
\hline$V_{M 3}$ & 20 \\
\hline$k$ & 0.2 \\
\hline$k_{f}$ & 0.04 \\
\hline$K_{2}$ & 1 \\
\hline$K_{R}$ & 2 \\
\hline$K_{A}$ & 0.9 \\
\hline$m$ & 2 \\
\hline$n$ & 2 \\
\hline$p$ & 4 \\
\hline
\end{tabular}

Table 2. Parameter values used in figure 31.

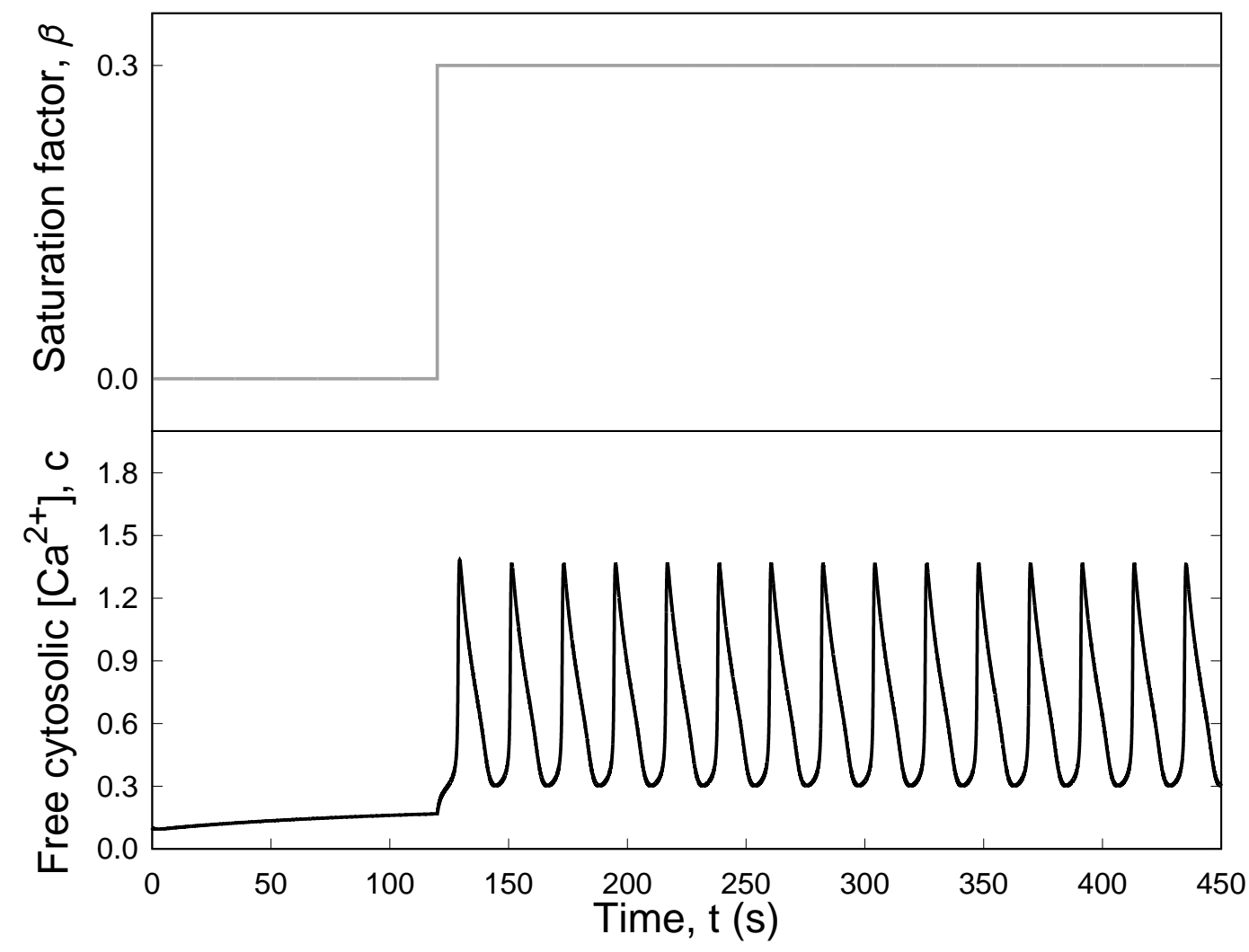

Figure 31. Simulation of the minimal model for $\mathrm{Ca}^{2+}$ oscillations as a match to Clark result in figure 17. Parameter values used for this simulation are shown in table 2.

The result shown in figure 31 above shows that the oscillation frequency of ambient temperature may be encoded by AFD calcium oscillations that are controlled by an absolute level of the saturation factor $\beta$, in this model. 


\section{APPENDIX C: FAST INACTIVATION POTASSIUM CURRENT MODEL}

The equations stated below comprise a model of a patch of neuronal membrane, and are taken from the book by Izhikevich [22].

$$
\begin{aligned}
C \frac{d V}{d t} & =I-g_{L}\left(V-V_{L}\right)-g_{K} m h_{\infty}(V)\left(V-V_{K}\right) \\
\frac{d m}{d t} & =\frac{m_{\infty}(V)-m}{\tau_{m}} \\
m_{\infty}(V) & =\frac{1}{1+\exp \left[\left(V-V_{1 / 2}^{m}\right) / k_{m}\right]} \\
h_{\infty}(V) & =\frac{1}{1+\exp \left[\left(V_{1 / 2}^{h}-V\right) / k_{h}\right]}
\end{aligned}
$$

where $t$ is time in milliseconds. $\exp (x)$ denotes the exponential function $e^{x}$, such that $\frac{d}{d x}\left(e^{x}\right)=e^{x}$. $C$ is the membrane capacitance per unit area $\left(\mu \mathrm{F} / \mathrm{cm}^{2}\right) . V$ is the membrane voltage $(\mathrm{mV}) . I$ is the applied current per unit area $\left(\mu \mathrm{A} / \mathrm{cm}^{2}\right) \cdot g_{L}$ and $g_{K}$ are the maximum conductances per unit area $\left(\mathrm{mS} / \mathrm{cm}^{2}\right)$ for the leak channels $(\mathrm{L})$ and the potassium channels $(\mathrm{K})$, respectively. $V_{L}$ and $V_{K}$ are the Nernst potentials for the leak and potassium currents, and are taken to be $-60 \mathrm{mV}$ and $-80 \mathrm{mV}$, respectively. $m$ is the time-dependent potassium activation, $m_{\infty}(V)$ is the voltagedependent steady-state potassium activation, and $h_{\infty}(V)$ is the voltage-dependent steady-state potassium inactivation which is reached instantaneously. $V_{1 / 2}^{m}$ is the half-activation voltage with slope $k_{m}$ and $V_{1 / 2}^{h}$ is the half-inactivation voltage with slope $k_{h}$. The symbols " $m$ " and " $h$ " are superscripts in the half-activation voltages, NOT exponents. For parameter values, see table 3.

\begin{tabular}{|c|c|}
\hline Parameter & Value \\
\hline$g_{L}$ & $0.2 \mathrm{mS} / \mathrm{cm}^{2}$ \\
\hline$g_{K}$ & $5.0 \mathrm{mS} / \mathrm{cm}^{2}$ \\
\hline$V_{1 / 2}^{m}$ & $-30 \mathrm{mV}$ \\
\hline$k_{m}$ & $10 \mathrm{mV}$ \\
\hline$V_{1 / 2}^{h}$ & $-70 \mathrm{mV}$ \\
\hline$k_{h}$ & $-11 \mathrm{mV}$ \\
\hline$\tau_{m}$ & $20 \mathrm{~ms}$ \\
\hline$V_{K}$ & $-80 \mathrm{mV}$ \\
\hline$V_{L}$ & $-60 \mathrm{mV}$ \\
\hline
\end{tabular}

Table 3. Parameter values used in figure 32 . 


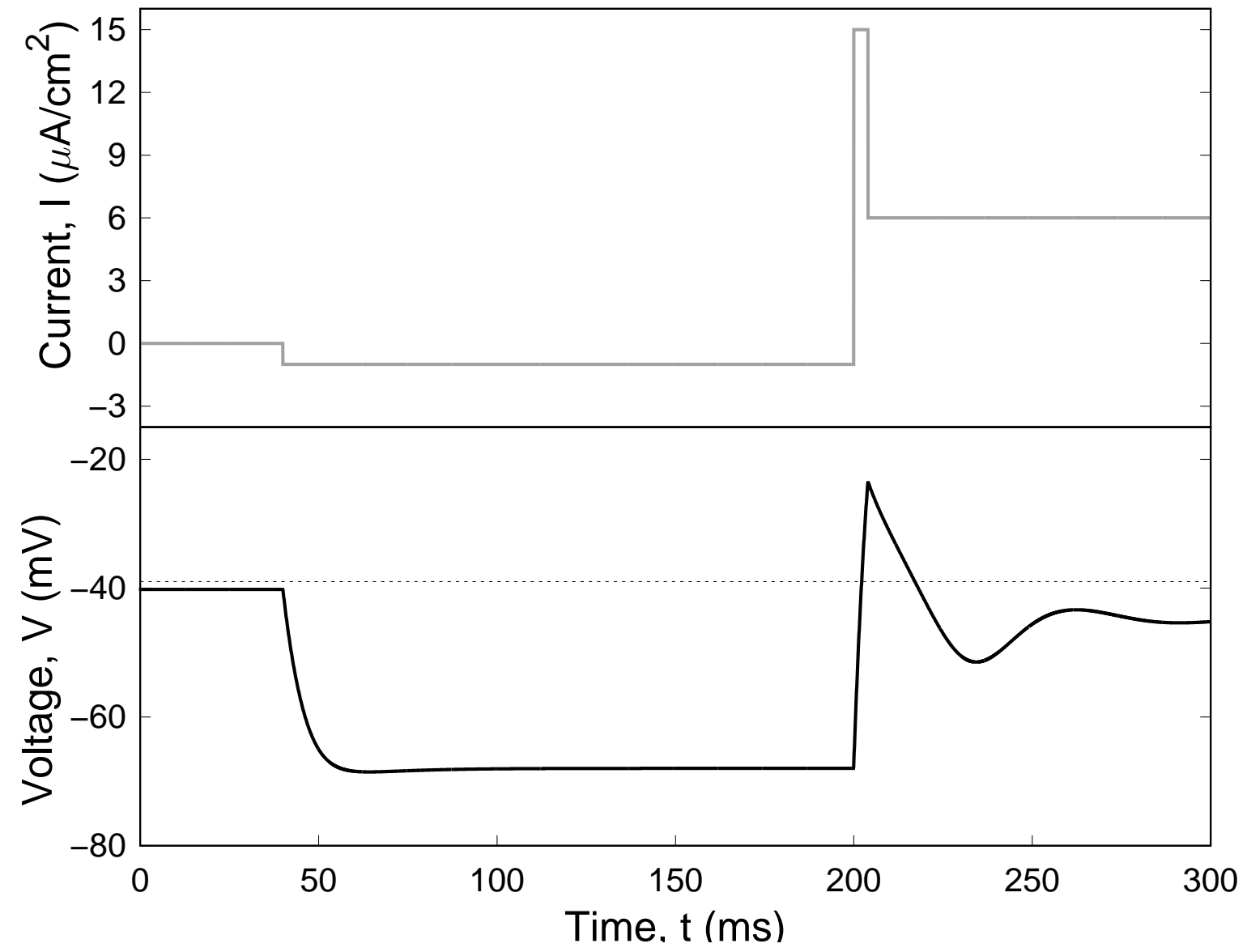

Figure 32. Simulation of equations 30-33 for a particular current stimulus, $I$, to serve as a comparison to experimental results from [32] shown in figure 33. Horizontal dotted line at $V=-40 \mathrm{mV}$ added for reference. 

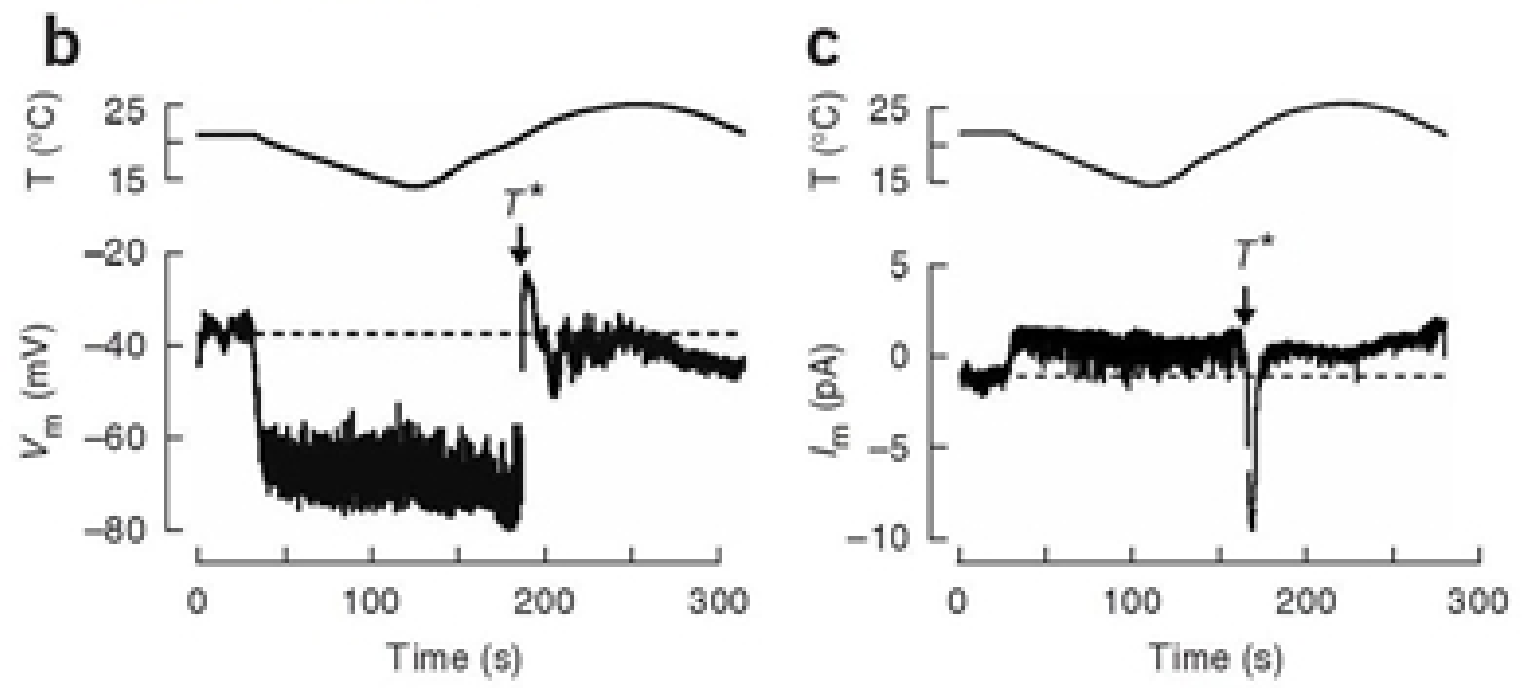

Figure 33. Experimental data from figure 1 of Ramot et al. (2008). Reprinted with permission from Springer: Nature Neuroscience [33] Copyright 2008.

Disregarding the clear difference in noise levels between low and high temperatures, there is a slight mismatch between the current in the experimental plot in figure 33 (right) and the theoretical current in 32 (top) which was manually chosen to acquire the theoretical voltage response in figure 32 (bottom). In the experiment, the membrane current $I_{m}$ returns to its original average level after the downward spike at $t \approx 170 \mathrm{~s}$. The theoretical neuron, on the other hand, requires that the current return to a higher baseline after the large spike at $t \approx 200 \mathrm{~s}$ in order to display the correct voltage response. This shows that a model with purely voltage-dependent currents cannot account for the response of AFD, which justifies the use of a $c$-gated $\mathrm{K}^{+}$current in the model of chapter III. 


\section{APPENDIX D: NUMERICAL DETAILS AND STATEMENT OF ACCESS}

All computational models mentioned here have been implemented in the programming language

$\mathrm{C}++$ on an HP laptop computer with Intel core i7 processor using the fourth-order Runge-Kutta algorithm. The codes that produced the figures involving simulations in this report are available upon request. If interested, please contact ZDM428@gmail.com. 